\title{
Le pouvoir des mots au Moyen Âge
}

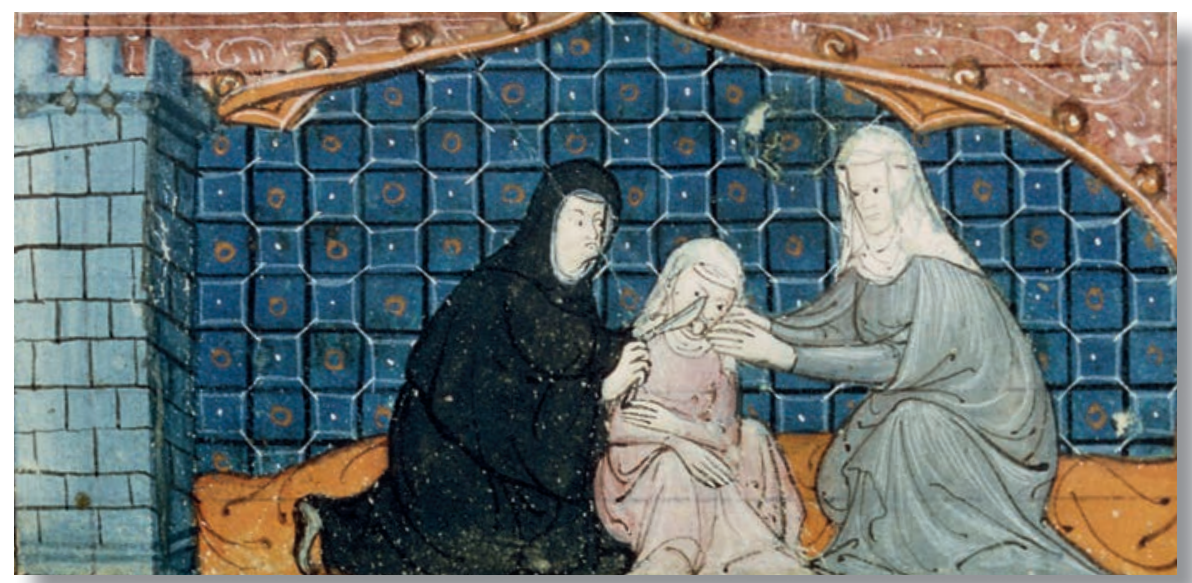

Études réunies par

Nicole BÉRIOU, Jean-Patrice BOUDET et Irène ROSIER-CATACH

\section{BREPOLS}




\title{
Lucie DolEŽAlová et Farkas Gábor KIss
}

\section{LE POUVOIR DES MOTS DANS L'ART DE LA MÉMOIRE À LA FIN DU MOYEN ÂGE*}

\begin{abstract}
On appelle 'soustraction des choses' (subtractio ex re) le fait de soustraire quelque chose du sujet que l'on veut imaginer et situer dans un lieu. Par exemple, si l'on soustrait le début d'une chose, on l'associe à la soustraction du début d'un mot, si l'on soustrait le milieu d'une chose, on supprime une syllabe au milieu du mot, et de même pour la fin. Par exemple, si tu veux mémoriser le mot 'Ismaël', il faut que tu imagines un chien (canIS) dont la moitié antérieure est écorchée, et que tu ajoutes du miel (MEL) à l'image, comme si le chien le mangeait ${ }^{1}$.
\end{abstract}

C'est le patricien vénitien Leonardo Giustiniani, humaniste et poète (1388-1446), qui conseille cette technique de mémorisation au moyen de cette image audacieuse et violente raccordant les éléments de deux mots (canis, mel) sur le plan imaginatif pour renforcer la mémoire d'un troisième (Ismael). Selon les règles de Giustiniani, on peut réorganiser les éléments des mots par d'autres méthodes analogues: ainsi l'addition de «cire» (cera) et d'«ailes » (ale) rappelle le mot céréale (cereale), et la transposition des syllabes (par exemple pastu pour stupa ou estalum pour mustela) aide particulièrement à se souvenir des mots étrangers et inconnus.

L'utilisation du pouvoir inhérent à la forme phonétique ou au contenu sémantique des mots était l'un des modes les plus importants et les plus efficaces de mémorisation à la fin du Moyen Âge, en vertu de la similitude fondamentale entre le fonctionnement de la mémoire et du langage. La

Les recherches à l'origine de cet article ont été subventionnées par Le Ministère de l'Éducation, de la Jeunesse et des Sports (Institutional Support for Longterm Development of Research Organizations), Université Caroline de Prague, Faculté des Sciences Humaines, et par une bourse de la fondation Bolyai de l'Académie Hongroise des Sciences. Nous remercions Benoît Grévin pour son aide dans la révision de cet article.

1 «Ex re autem subtractio est, per quam rei imaginatae et in loco positae aliquid detrahimus: ut si detrahamus principium rei, intelligamus detrahi principium diccionis; si medium, medium; si finem, finem. Quod genus si hoc nomen Hismael velis tenere, constitue canem qui ex parte anteriori ad medium sit expers pellis, hinc deinde subice mel, quasi ut comedat. » A. Oberdorfer, «Le 'Regulae artificialis memoriae' di Leonardo Giustiniani», Giornale storico della letteratura italiana, 60 (1912), p. 117-127, ici 122-123. Sur l'auteur, cfr Sabine Seelbach, Ars und Scientia. Genese, Überlieferung und Funktionen der mnemotechnischen Traktatliteratur im 15. Jahrhundert. Mit Edition und Untersuchung dreier deutscher Traktate und ihrer lateinischen Vorlagen, Tübingen, 2000, p. 38-40 et 507-508. 
première et la plus générale des catégories que Giustiniani et d'autres auteurs ${ }^{2}$ utilisent pour décrire le processus de génération de ces symboles mnémoniques artificiels est l'impositio, un terme technique de la linguistique médiévale désignant l'opération d'imposition, c'est-à-dire d'institution volontaire du signe, par lequel un son vocal signifiant était associé à un signifié3. C'était par «imposition » qu'il fallait préparer un dictionnaire mental personnalisé, constitué de symboles fixes et s'étendant aux sujets essentiels de la vie, comme les noms des mois, les nombres ou les unités monétaires. Tout comme le langage utilise l'opération d'imposition pour établir des liens entre les mots et les choses, les signes et les signifiés, de même la mémoire artificielle évoque un souvenir à l'aide des rapports artificiellement générés entre un symbole mnémonique et l'objet mémorisé. L'étroitesse de cette relation entre le langage et la mémoire était également claire pour les théoriciens médiévaux de l'art de la mémoire, lesquels faisaient d'ailleurs fréquemment appel à la similitude de la mémorisation et de l'écriture.

Tout comme ceux qui connaissent l'écriture peuvent écrire ce qui a été dit, et prononcer ce qui a été écrit, de même ceux qui ont étudié l'art mnémotechnique peuvent retenir les choses exprimées par les lieux mnémoniques et parler par cœur sur la base des lieux (loci) .

Dans les pages suivantes, nous tenterons d'examiner le rôle des mots dans l'art de la mémoire médiévale à plusieurs niveaux, et de livrer un début d'explication au phénomène que représenta l'art de la mémoire dans les derniers siècles du Moyen Âge. Comment un système fondé sur la reproduction de la relation sémantique existant dans le langage, si arbitraire et si redondante à première vue, a-t-il pu devenir si populaire parmi les intellectuels de la fin du Moyen Âge?

Les études sur la mémoire ont joui ces dernières décennies d'une attention inédite, en particulier dans les sciences cognitives, mais aussi en histoire en général ${ }^{5}$, et plus particulièrement dans les recherches portant sur

2 Par exemple Lodovico da Pirano: cfr B. Ziliotto, «Frate Lodovico da Pirano e le sue Regulae memoriae artificialis», Atti e memorie della Società Istriana di Archeologia e Storia Patria, 49 (1937), p. 215.

3 Sur l'impositio, cfr I. Rosier-Catach, La parole comme acte. Sur la grammaire et la sémantique au XIII siècle, Paris, 1993, p. 117-142. Pour les débats sur l'arbitrarité de l'imposition, v. I. RosierCatach, La parole efficace. Signe, rituel, sacré, Paris, 2004, 168-171.

4 B. Ziliotto, «Frate Lodovico da Pirano», p. 217. Cette comparaison (bien connue depuis le Phèdre de Platon) est omniprésente dans les traités sur l'art de mémoire à la fin du Moyen Âge.

5 Voir par exemple A. Assmann, Erinnerungsräume. Formen und Wandlungen Kulturellen Gedächtnisses, Munich, 1999; J. Assmann, "Collective Memory and Cultural Identity», New German Critique, 65 (1995), p. 125-133. 
le Moyen Âge ${ }^{6}$. Tandis que les sciences cognitives et la neuropsychologie se concentrent avant tout sur les mystères concernant le fonctionnement de la mémoire humaine, l'histoire tend à délaisser l'effort d'une reconstruction précise du déroulement des événements passés. Cette reconstruction a été remplacée par des recherches sur la manière dont la «mémoire» de ces événements est créée et construite, c'est-à-dire sur la manière dont les événements sont présentés par les contemporains. On tente d'observer quelles informations ces derniers mettent en relief et ce qu'ils taisent, quelle image de l'histoire ils créent et quels sont les modes de transmission, modification et correction de cette image.

Dans le domaine de l'histoire littéraire, le livre de la chercheuse américaine Mary Carruthers, The Book of Memory ${ }^{7}$, est celui qui a bouleversé l'appréhension du rôle de la mémoire au Moyen Âge. Carruthers démontre que l'homme médiéval concevait la mémoire comme une partie essentielle du processus créatif, pratiquement comme l'équivalent de ce que représente de nos jours l'imagination. La mémoire n'était pas pour lui seulement passive et imprévisible, les souvenirs n'étaient pas que des objets entreposés que l'on peut reprendre n'importe quand, sous une forme inchangée. Une mémoire bien structurée était le signe d'une bonne compréhension. En effet, l'intelligence était l'aptitude à s'orienter dans la mémoire et à relier des informations données. La remémoration formait donc un processus créatif au cours duquel les souvenirs étaient à chaque fois recréés ${ }^{8}$. Cette importance qu'avait la mémoire dans les théories psychologiques médiévales explique tout naturellement l'intérêt porté à la maîtrise de la mémoire.

C'est en particulier au Moyen Âge tardif que des traités sur l'art de la mémoire, artes memoriae ou artes memorativae, se répandent. Ces textes sortent du cadre de l'art de la mémoire classique, tel qu'il avait été établi en tant que partie de la rhétorique et dont les rhéteurs antiques se servaient lors de la rédaction des discours publics. C'est dans le troisième livre du traité anonyme

6 Voir par exemple P. J. Geary, Phantoms of Remembrance: Memory and Oblivion at the End of the First Millennium, Princeton, 1994; J. Coleman, Ancient and Medieval Memories. Studies in the Reconstruction of the Past, Cambridge, 1992; M. T. Clanchy, From Memory to Written Record: England 1066-1307, Oxford, 1993.

7 M. Carruthers, The Book of Memory. A Study of Memory in Medieval Culture, Cambridge, 1993; Eadem, The Craft of Thought: Meditation, Rhetoric, and the Making of Images 400-1200, Cambridge, 1998; Eadem et J. Ziolkowski, The Medieval Craft of Memory. An Anthology of Texts and Pictures, Philadelphia, 2003. En même temps d'importantes recherches ont été menées sur le lien entre la mémoire, l'intelligence et le langage mental dans la philosophie médiévale à partir d'Augustin: Cl. Panaccio, Le discours intérieur. De Platon à Guillaume d'Ockham, Paris, 1999, p. 108-119. Plus récemment, Olivier Boulnois a souligné l'importance des «images mentales» comme fondation anthropologique de l'interprétation de l'art médiéval: O. Boulnois, Au-delà de l'image. Une archéologie du visuel au Moyen Âge, Ve-Xvie siècle, Paris, 2008.

8 M. Carruthers, The Book of Memory, p. 1-15 et 46-79. 
«Rhétorique à Herennius » (Rhetorica ad Herennium), datant du $\mathrm{I}^{\mathrm{er}}$ siècle avant Jésus Christ, et que le Moyen Âge attribuait à Cicéron ${ }^{9}$, que cet art est décrit le plus précisément. On y trouve des règles détaillées, de même que la description des débuts mythiques de cet art: l'orateur Simonide avait été appelé hors d'une salle de banquet et pendant qu'il dialoguait à l'extérieur, les autres participants avaient été ensevelis, par suite de l'effondrement du toit. Simonide s'était rappelé plus tard où les différents participants avaient été assis et il avait pu identifier leurs corps en parcourant physiquement les décombres autour de la table.

L'art de la mémoire antique est donc fondé sur le système des lieux, loci, que l'orateur crée dans son imagination et où il dépose des images, imagines. En prononçant son propre discours, l'orateur parcourt de nouveau en imagination les lieux choisis et visualise les images qui y sont déposées. Ainsi surgissent dans sa mémoire les thèmes et les mots-clés du discours qu'il a préparé à l'avance. Les lieux peuvent être des édifices existants, bien connus de l'orateur, comme l'école ou la colonnade, ou bien des lieux imaginaires, mais classés par catégories et divisés en plus petits segments. En effet, les images sont souvent composées de nombreux éléments, tout en satisfaisant à la nécessité d'être embrassées d'un seul regard, d'être expressives et surprenantes. Elles doivent également être créées avec soin et d'une manière adaptée aux objectifs précis d'une personne concrète. Il s'agit d'imagines agentes, c'est-à-dire que les images sont tenues de "faire» quelque chose, d'éveiller le souvenir de la notion conservée. L'art de la mémoire est donc une stratégie générale qui est toujours adaptée aux circonstances concrètes.

Les images devront donc être choisies dans le genre qui peut rester le plus longtemps gravé dans la mémoire. Ce sera le cas, si nous établissons des similitudes aussi frappantes que possible; si nous prenons des images qui ne soient ni nombreuses ni floues, mais qui aient une valeur; si nous leur attribuons une beauté exceptionnelle ou une insigne laideur; si nous ornons certaines, comme qui dirait, de couronnes ou d'une robe de pourpre, pour que nous reconnaissions plus facilement la ressemblance, ou si nous les enlaidissons de quelque manière, en nous représentant telle d'entre elles sanglante, couverte de boue, ou enduite de vermillon, pour que la forme nous frappe davantage, ou encore en attribuant à certaines images quelque chose qui soulève le rire: car c'est là aussi un moyen pour nous de retenir plus facilement ${ }^{10}$.

9 Ad C. Herennium: De ratione dicendi (Rhetorica ad Herennium), éd. et trad. angl. H. Caplan, London, 1954, ou: Rhétorique à Herennius. De ratione dicendi ad C. Herennium, éd. G. Achard, Paris, 1989, http://www.mediterranees.net/art_antique/rhetorique/herennius/livre_3. html.

10 Ad Her III, 37, 22. 
La mémoire, memoria, se divise en memoria verborum et memoria rerum ${ }^{11}$. La mémoire des mots est en effet l'art de mémoriser un texte mot par mot. Il ne s'agit pourtant pas d'apprendre le texte par cœur: chaque mot, voire chaque syllabe est ici représenté par sa propre image. Dans le cas de la memoria rerum, ce sont les images de concepts entiers, de thèmes et de points d'appui du discours qui sont créées. Le discours est ultérieurement reconstruit sur la base de ces images, lors de son énonciation ${ }^{12}$.

Au Moyen Âge, le rôle de l'art de la mémoire antique change. En premier lieu, cet art apparaît en rapport étroit avec la méditation et la confession, ce qui le lie incontestablement à l'éthique et à la morale. Hugues de Saint-Victor recommande comme locus de base pour organiser les images mémorielles l'arche de Noé$^{13}$. L'art de la mémoire est lié aux structures bibliques connues ou aux bâtiments associés au christianisme de diverses manières ${ }^{14}$. Mais c'est au $\mathrm{Xv}^{\mathrm{e}}$ siècle que commence une véritable explosion du genre, explosion dont la genèse n'a jusqu'à présent pas été expliquée de manière convaincante.

Frances A. Yates a tenté d'expliquer la floraison soudaine de ce type de littérature par le changement qui affecte la notion de mémoire dans la Summa theologiae de saint Thomas d'Aquin. En exposant le système des vertus dans la Summa theologiae sur la base de l'éthique aristotélicienne, Thomas avait complété les éléments traditionnellement attachés à la vertu de prudence par la mémoire, en renforçant cette association à l'aide d'une définition empruntée à la partie mnémotechnique de l'Ad Herennium ${ }^{15}$. C'est ainsi que, selon Yates, la mémoire, qui était précédemment considérée comme un sujet rhétorique, aurait gagné en importance, étant dès lors considérée comme une composante de l'éthique, et soutenue par l'autorité de Thomas d'Aquin.

Dans l'état actuel de nos connaissances, c'est toutefois seulement au $\mathrm{Xv}^{\mathrm{e}}$ siècle, et non au $\mathrm{XIII}^{\mathrm{e}}$ ou au $\mathrm{XIV}^{\mathrm{e}}$ siècle que ce type de traité prolifère, pour devenir omniprésent dans presque toute l'Europe. D'importants manuscrits datant des deux siècles et demi séparant la mort de Thomas d'Aquin du début du $x^{e}$ siècle survivent, mais leur popularité est réduite, en comparaison des

11 «Comme les images doivent ressembler aux objets, nous devons, nous-mêmes, tirer de tous les objets des ressemblances. Les ressemblances doivent donc être de deux espèces, celles des choses et celles des mots. Les ressemblances des choses se produisent, lorsque nous évoquons une image générale des objets pris en eux-mêmes; les ressemblances de mots s'établissent, lorsqu'on note par une image le souvenir de chaque mot et de chaque terme » (Ad Her III, 20).

12 Ad Her III, 16-24.

13 De arca Noe mystica dans PL 176, col. 681-703; édition critique par P. Sicard, «Libellus de formatione arche", dans Hugo de Sancto Victore, De archa Noe pro archa sapientie cum archa Ecclesie et archa matris gratie. Libellus de formatione arche Turnhout, 2001 (CCCM 176), p. 119-162; Id., Diagrammes médiévaux et exégèse visuelle. Le Libellus de formatione arche de Hugues de SaintVictor, Turnhout, 1993.

14 Cfr M. Carruthers, The Craft of Thought, p. 221-276.

15 F. A. Yates, The Art of Memory, Chicago, 1966, p. 73-76 et 82-83. 
cinquante-six traités que Sabine Seelbach a trouvés en limitant sa recherche à la seule tradition des arts de la mémoire datant $d u x^{e}$ siècle. Considérés dans leur ensemble, ces traités tardifs survivent dans plus de deux-cent cinquante manuscrits et au moins quinze éditions incunables ${ }^{16}$.

Il y a probablement plusieurs raisons à cette popularité inattendue de l'art de la mémoire. Vers 1400, le nombre des universités ${ }^{17}$, et par conséquent des étudiants, avait considérablement augmenté. Au fur et à mesure que le besoin de classer et d'organiser les informations se faisait plus pressant, tout un ensemble de stratégies mnémoniques commencèrent à être activement utilisées $^{18}$. Il ne semble pas que l'art de la mémoire ait jamais été inclus dans les cursus officiels: il n'était enseigné qu'en privé, avec d'autres disciplines pratiques comme l'art épistolaire (ars epistolandi), l'algorismus ou l'arbor consanguinitatis et affinitatis. L'accroissement du nombre des étudiants suscita un intérêt accru pour l'enseignement de ces disciplines subsidiaires, et par suite assura à ceux qui le délivraient des revenus satisfaisants. Ces professeurs voyageaient entre les universités de l'Europe entière. Nous pouvons mentionner à titre d'exemple Jacobus Publicius, Conrad Celtis, Thomas Murner et Johannes Cusanus ${ }^{19}$. Beaucoup de manuscrits contenant des traités sur l'art de la mémoire proviennent des milieux universitaires et y sont étroitement liés. On y commentait des traités antiques sur la rhétorique et la poétique, et c'est pourquoi l'on y prêtait une attention plus détaillée qu'auparavant aux traités de Cicéron et Quintilien, sections consacrées à l'art de mémoire comprises. L'étude de l'Institutio oratoria de Quintilien recommença

16 Nous devrions ajouter à ce nombre les copies de la Rhétorique à Herennius, qui mettaient à disposition les mêmes enseignements mnémotechniques, mais dans ce dernier cas, le témoignage n'est bien sûr pas univoque, car les copistes et propriétaires de ce traité n'étaient pas nécessairement intéressés par l'art de la mémoire. Voir The Rhetoric of Cicero in its Medieval and Early Renaissance Commentary Tradition, éd. V. Cox et J. Ward, Leiden, 2006.

17 En Allemagne, de nouvelles universités sont alors établies par une succession rapide de fondations: Heidelberg 1386, Cologne 1388, Erfurt 1392, Leipzig 1409, Rostock 1419, Greifswald 1456, Fribourg 1457, Ingolstadt 1472, Mayence 1477, Tübingen 1477, Francfort-surl'Oder 1506.

18 Les condensations et résumés (à la fois textuels et visuels) de la Bible, des Sentences et du droit canon étaient très populaires à cette époque. Voir S. Rischpler, «Cœur voyant. Mémoriser les Sentences de Pierre Lombard», dans Medieval Memory. Image and Text, éd. Fr. Willaert et al., Turnhout, 2004, p. 3-40; L. Doležalová, «Mémoriser la Bible au bas Moyen Âge? Le Summarium Biblicum aux frontières de l'intelligibilité», Cahiers électroniques d'histoire textuelle du LAMOP, 3 (2010), p. 1-45.

19 Voir A. Sottili, Giacomo Publicio, 'Hispanus' e la diffusione dell'Umanesimo in Germania, Barcelona, 1985 ; S. Heimann-Seelbach, Ars und scientia. Genese, Überlieferung und Funktionen der mnemotechnischen Traktatliteratur des 15. Jahrhunderts, Tübingen, 2000, p. 133-140 ; R. Wójcik, «Straßburg - Freiburg - Paris - Krakau. Zu den möglichen Inspirationsquellen Thomas Murners, des Autors des Chartiludium logicae sive logica memorativa (1507/1509)", dans Daphnis. Zeitschrift für Mittlere Deutsche Literatur und Kultur der Frühen Neuzeit, 40 (2011), p. 63-88; et sur la vie de Johannes Cusanus notre anthologie en préparation: The Art of Memory in East Central Europe in the late Middle Ages. 
ainsi après une longue interruption, après que Poggio Bracciolini l'eut redécouverte dans l'abbaye de Saint-Gall, en $1416^{20}$.

Un grand nombre de traités sur l'art de la mémoire proviennent des milieux franciscains, probablement par suite du développement de la nouvelle technique de prédication qui y avait pris naissance, en particulier parmi les Observants $^{21}$. Ce nouveau type de sermon avait été introduit par Saint Bernardin de Sienne qui avait lui-même recours à différents moyens rhétoriques pour influencer les auditeurs dans un style très affecté et émotif, et se réfère plusieurs fois à l'art de la mémoire ${ }^{22}$. Il est à l'origine d'une école de prédication (Jean Capistran, Roberto Caracciolo da Lecce, Jacopo delle Marche, etc. $)^{23}$. Celle-ci se répandit rapidement en Europe centrale, quand des prédicateurs italiens vinrent prêcher contre les hérétiques, les Juifs et les Turcs. Presque tous les traités sur l'art de mémoire provenant de Pologne peuvent ainsi être associés aux Observants (Stanislaw Korzybski, Paulinus de Sklabmierz, Jan Szklarek, Antoni de Radomsko) ${ }^{24}$.

Il est enfin peut-être possible de relier l'art de la mémoire à la réforme monastique de la dévotion et au mouvement de la devotio moderna, ainsi qu'aux changements radicaux qui affectèrent la culture visuelle durant le Moyen Âge tardif. Typique de l'art chrétien du Moyen Âge tardif est la recherche d'une combinaison de différents facteurs sensoriels, susceptibles de donner une impression comparable aux imagines agentes de l'art de la mémoire. Or il est conseillé d'utiliser des images lors de la prière ou de la méditation. On trouve ainsi des modes d'emploi détaillés expliquant comment contempler de manière systématique et organisée différentes parties des représentations pieuses (par exemple les arma Christi ou les sept Plaies de Jésus) pendant la prière. Ces instructions sont dans leur principe similaires aux conseils contenus dans les traités sur l'art de la mémoire. Qui plus est, la

20 R. Sabbadini, Le scoperte dei codici latini e greci ne'secoli XIV e XV, t. II, Firenze, 1905, p. $247-248$. L. D. Reynolds, ed., Texts and Transmission: A Survey of the Latin Classics, Oxford, 1983, p. 332334 ; M. Winterbottom, "Fifteenth Century Manuscripts of Quintilian », Classical Quarterly, 2: 17 (1967), p. 339-369; F. Murru, «Poggio Bracciolini e la riscoperta dell'Institutio oratoria del Quintiliano ", Critica storica, 20 (1983), p. 621-626.

21 K. Rivers, Preaching the Memory of Virtue and Vice. Memory, Images, and Preaching in the Late Middle Ages, Turnhout, 2011.

22 C. Delcorno, «L'ars praedicandi di Bernardino da Siena », Lettere italiane, 32 (1980), p. 453-454.

23 A. Ghinato, "La predicazione francescana nella vita religiosa e sociale del Quattrocento", Picenum Seraphicum, 10 (1973), p. 24-94.

24 Voir R. Wójcik, 'Opusculum de arte memorativa' Jana Szklarka. Bernardyński traktat mnemotechniczny z 1504 roku (Prace Biblioteki Uniwersyteckiej 28), Poznań, 2006; Id., éd., Culture of Memory in East Central Europe in the Late Middle Ages and Early Modern Period. Conference Proceedings - Ciążeń, March 12-14, 2008, Poznań, 2008; Id., "Populus meus captivus ductus est: On the Polish Franciscan's Mnemonic Treatise form the Fifteenth Century», dans Strategies of Remembrance: From Pindar to Hölderlin, éd. L. Doležalová, Newcastle upon Tyne, 2009, p. $175-184$. 
Passion du Christ est alors représentée de manière naturaliste, avec un accent particulier sur la douleur physique, évoquant ainsi les images mnémoniques, dans lesquelles on recommande également d'utiliser la violence et le sang ${ }^{25}$.

Or il faut souligner que l'art de la mémoire médiéval n'était pas seulement une méthode pour améliorer la mémoire appliquée, mais qu'il recouvrait des préoccupations bien plus vastes. Des conseils pour améliorer la mémoire apparaissent souvent dans les traités médicaux, et incluent des prescriptions et des avis de bon sens tels que la nécessité de dormir suffisamment, de ne pas travailler trop durement, de se concentrer sur un objet à la fois, etc. Il faut mettre ces témoignages en relation avec le grand nombre de traités sur l'art de mémoire. Ils attestent la mise en pratique de cet art, et tout autant sa transformation.

Pris dans leur ensemble, les traités sur l'art de la mémoire sont en beaucoup d'aspects similaires. Ils rappellent généralement les règles de base concernant l'art de la mémoire, et divergent essentiellement par le choix des exemples de lieux et d'images qu'ils proposent. La raison de cette divergence est simple: l'un des théorèmes essentiels de l'art de mémoire est que chacun doit choisir les lieux et les images à sa convenance, car ce qui aide la mémoire de l'un peut encombrer la mémoire de l'autre ${ }^{26}$. Aussi chaque auteur de traité sur la mémoire fournit-il ses propres suggestions de lieux et d'images mnémotechniques, afin de les suggérer, plutôt que de les imposer au lecteur.

Focalisons-nous à présent, dans le cadre du système des lieux et des images, sur la mémoire verbale, pour montrer la place du pouvoir des mots dans cet art spécifique. Car en dépit des apparences, l'art de la mémoire est bien loin de ne fonctionner qu'avec la mémoire visuelle. Au contraire, la relation entre le visuel et le verbal est dans ce domaine des plus complexes et digne d'attention. Les deux champs sont étroitement connectés. Les paroles interviennent en effet dans la "peinture de l'imagination » à deux niveaux fondamentaux de l'art de la mémoire: dans la création des lieux, et dans celle des images.

\section{LES LIEUX DE LA MÉMOIRE: LE POUVOIR STRUCTUREL DES MOTS}

Les lieux de la mémoire servent de structure permanente, bâtie dans l'esprit pour être occupée par des images temporaires. L'auteur de l'Ad Herennium explique à leur propos:

25 P. Parshall, «The Art of Memory and the Passion», The Art Bulletin, 81:3 (1999), p. 456-472.

26 «Praeterea similitudine alia alius magis commovetur. Nam ut saepe, formam si quam similem cuipiam dixerimus esse, non omnes habemus adsensores, quod alii videtur aliud, item fit <in〉 imaginibus, ut, quae nobis diligenter notata sit, ea parum videatur insignis aliis. Quare sibi quemque suo commodo convenit imagines conparare» (Ad Her III, 23, 9). 
Locos appellamus eos, qui breviter, perfecte, insignite aut natura aut manu sunt absoluti, ut eos facile naturali memoria conprehendere et amplecti queamus: < ut a aedes, intercolumnium, angulum, fornicem et alia, quae his similia sunt. (Par cases/lieux, nous entendons les ouvrages de la nature ou de l'art tels que, dans un espace restreint, ils forment un tout complet et capable d'attirer l'attention, si bien que la mémoire naturelle puisse facilement les saisir et les embrasser: tels sont un palais, un entre-colonnement, un angle, une voûte et d'autres choses semblables) (Ad Her III, 16, 29).

Comme l'auteur de la Rhétorique Ad Herennium, les auteurs médiévaux partent de leur environnement, et proposent comme lieux de mémoire le couvent, l'église, l'école, le pré, etc.

Avec la prolifération des arts de la mémoire à la fin du Moyen Âge, de nouveaux loci sont également utilisés: on rencontre ainsi dans le traité de Jacobus Publicius des illustrations représentant un homme et une femme nus, sans aucun commentaire. Cette absence d'explicitation s'explique facilement: chaque enseignant de l'art de la mémoire désirait gagner sa vie, raison pour laquelle il aspirait à se rendre indispensable. Ces traités sont donc rédigés de sorte qu'ils restent presque incompréhensibles sans explication du professeur. Ce n'est que grâce à d'autres sources que nous savons que le corps humain (ou éventuellement animal) était en effet depuis peu utilisé comme lieu de la mémoire, au lieu du palais ou du couvent. Des images particulières étaient déposées près de la tête, de chaque bras, du ventre et de chaque jambe.

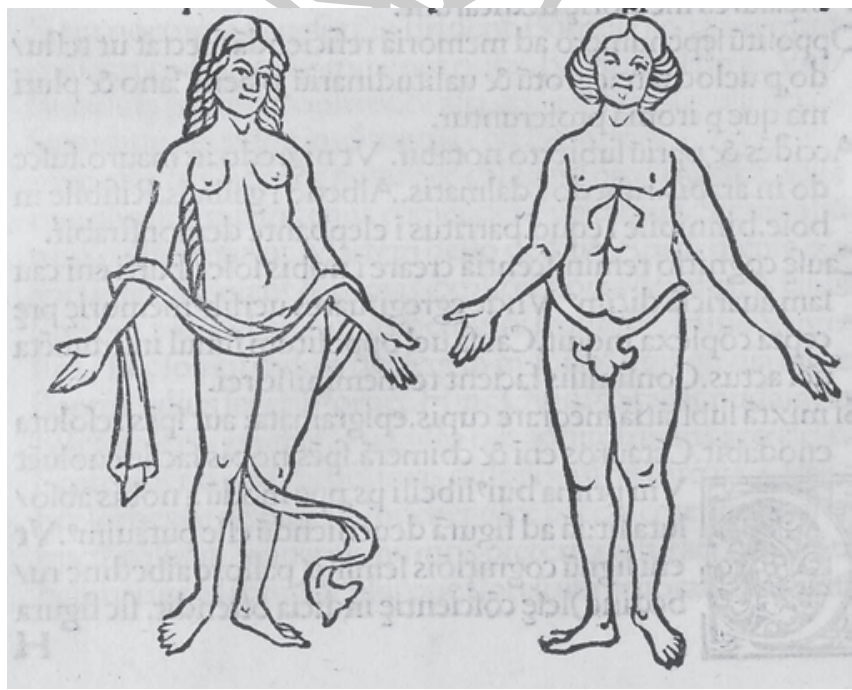

Fig. 1. Jacobus Publicius, Oratoriae artis epitoma... insuper et perquam facilis memoriae artis modus, Venise: Ratdolt, 1485, fol. H1v. 
Un exemple particulièrement répandu en Europe centrale est le Rationarium Evangelistarum qui était devenu un vrai best-seller. Il s'agit d'un outil pour mémoriser les Évangiles, où les images correspondant aux chapitres particuliers sont adaptées aux représentations d'un homme (Matthieu), d'un lion (Marc), d'un taureau (Luc) et d'un aigle (Jean) ${ }^{27}$. D'un manuscrit polonais proviennent les documents qui prouvent l'utilisation de représentations corporelles pour mémoriser des terminaisons latines. Nous y trouvons un homme dont la jambe est percée par une épée, une image «frappante» (Planche 1$)^{28}$. Des images de diables, grotesques, donc " mémorables », étaient utilisées pour aider à mémoriser un prêche: elles prouvent clairement l'usage actif de la méthode par les prédicateurs (Planche 2) ${ }^{29}$.

Mais les lieux ne consistent pas nécessairement en images seules: leur structure peut être complètement verbale. L'arrangement était déjà important pour l'auteur de l'Ad Herennium ${ }^{30}$. Le Moyen Âge tardif, plus obsédé encore par l'ordre, apporte des innovations en plus des lieux de mémoire purement visuels. Différents auteurs proposent un système lexical: l'étudiant doit apprendre 100 mots, divisés en groupes de cinq (un chiffre certainement en rapport avec les cinq doigts sur la main - autre lieu de mémoire fréquent) ${ }^{31}$ et il doit déposer les images particulières dans ces «lieux». Les 100 mots

27 Voir S. Rischpler, Biblia sacra figuris expressa. Mnemotechnische Bilderbibeln Des 15. Jahrhunderts (Wissensliteratur im Mittelalter 36), Wiesbaden, 2001. Pour une traduction anglaise et notes critiques, voir M. Carruthers et J. Ziolkowski, The Medieval Craft of Memory. An Anthology of Texts and Pictures, Philadelphia, 2003, p. 255-293.

28 F. G. Kiss, «Valentinus de Monteviridi (Grünberg) and the art of memory of Conrad Celtis», dans Culture of Memory in East Central Europe, ed. R. Wójcik, Poznań, 2008, p. 105-118.

29 Voir R. Wójcik, «Populus meus captivus ductus est», p. 175-184. Ces outils sont incontestablement similaires aux images du Christ et aux images médicales des lieux propices à faire une saignée. La saignée médiévale (sur laquelle nous ne pouvons pas nous appesantir dans ces pages) était liée à l'astrologie. La détermination des bons emplacements dépendait de la position des planètes. On rencontre même des outils qui combinent l'illustration des emplacements de la saignée et des signes du zodiaque.

30 «Item putamus oportere <ex ordine hos locos habere, > ne quando perturbatione ordinis inpediamur, quo setius, quoto quoquo loco libebit, vel ab superiore vel ab inferiore parte imagines sequi et ea, quae mandata locis erunt, edere possimus : nam ut, si in ordine stantes notos quomplures viderimus, nihil nostra intersit, utrum ab summo an ab imo an ab medio nomina eorum dicere incipiamus, item in locis ex ordine conlocatis eveniet, ut in quamlibebit partem quoque loco lubebit imaginibus commoniti dicere possimus id, quod locis mandaverimus» (De même, selon nous, il faut que nous ayons un ordre arrêté dans la disposition de ces cases, pour que leur confusion n'aille pas nous empêcher de suivre les images en quelque ordre qu'il nous plaira, en entamant la série par le commencement ou par la fin, non plus que d'exprimer ce que nous aurons confié aux différentes cases) (Ad Her III, 17, 30).

31 La division en groupes de cinq notions est déjà suggérée par l'auteur de l'Ad Herennium : «Et pour éviter toute erreur dans le nombre des cases, il faut donner un indice à tous les multiples de cinq; par exemple, si, à la cinquième, nous plaçons comme indice une main d'or, à la dixième (decimo) une de nos connaissances, dont le prénom sera Decimus, il sera facile en continuant la série, d'en faire autant pour tous les multiples de cinq» (Ad Her III, 18, 31). 
choisis forment une charpente organisée qui peut être réutilisée pour un autre dépôt d'informations.

Lors de la création de ces lieux, les auteurs utilisent souvent l'alphabet, grâce auquel il est possible d'identifier facilement la position de n'importe quel groupe de cinq termes. Ainsi, par exemple, le premier groupe de cinq mots peut contenir des ouvrages commençant par $\mathrm{Ba}, \mathrm{Be}, \mathrm{Bi}, \mathrm{Bo}, \mathrm{Bu}$, le second des titres commençant par $\mathrm{Ca}, \mathrm{Ce}, \mathrm{Ci}, \mathrm{Co}, \mathrm{Cu}$, etc., comme c'est le cas dans la Margarita philosophica de Gregor Reisch, datant de 1508. Mais d'autres arrangements sont également possibles. La liste de cent unités est souvent thématique, comme c'est le cas dans l'art de la mémoire de Mattheus Beran. Un exemple particulier est donné par un folio du manuscrit Prague, Bibliothèque nationale, I. G. 11a, dans lequel chaque groupe de cinq unités consiste en un animal, entouré par quatre artisans.

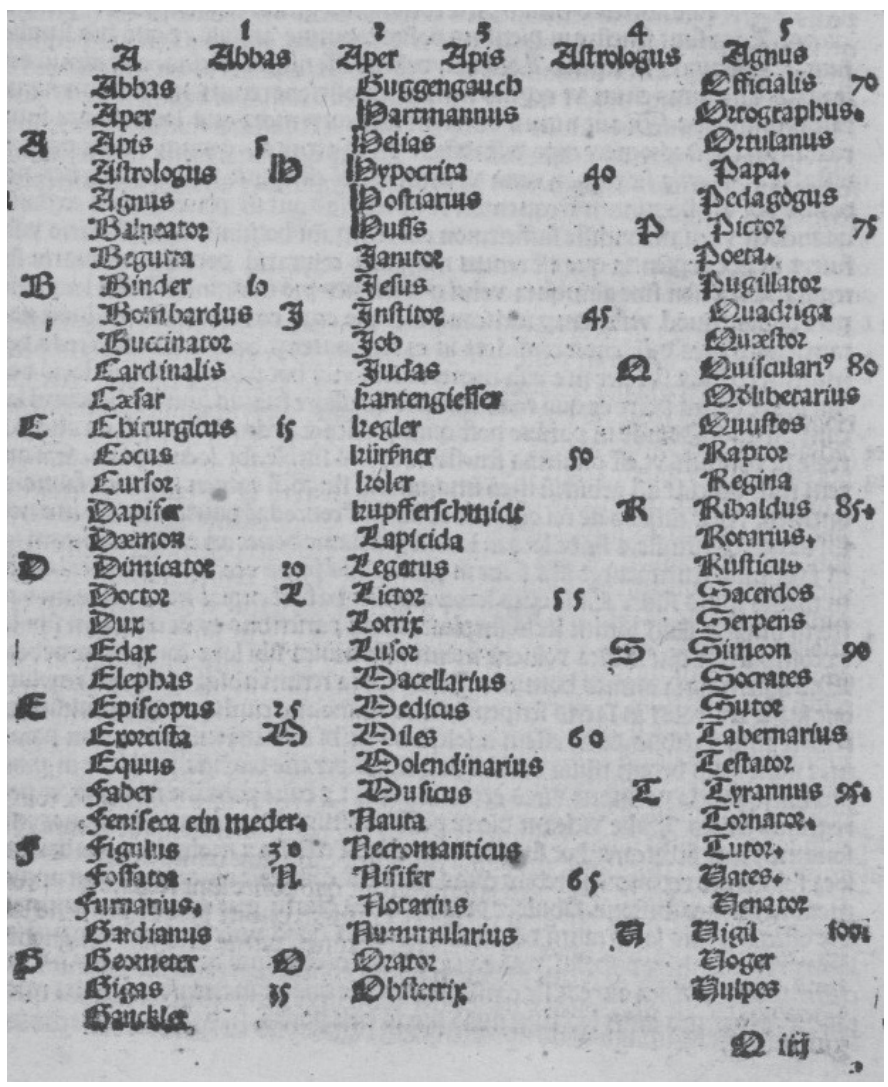

Fig. 2. Gregor Reisch, Margarita philosophica

Strasbourg, Grüninger, 1508, fol. Q4r 


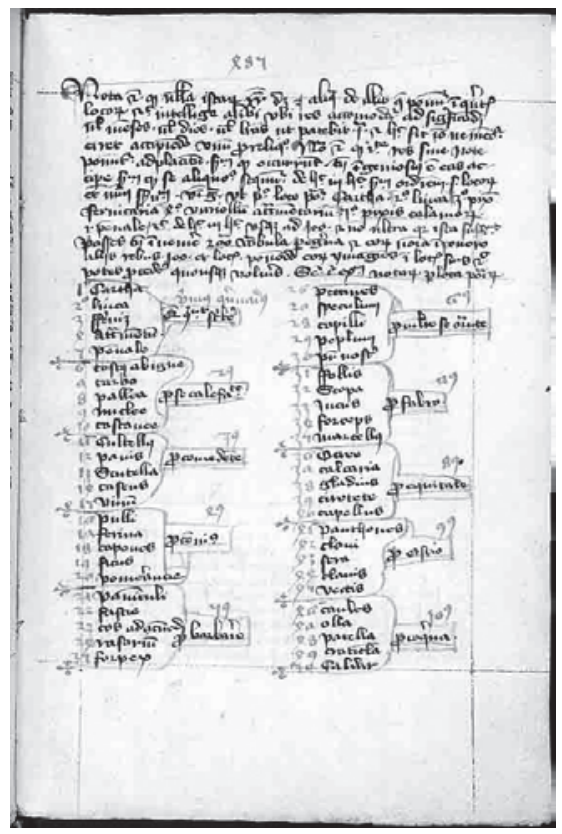

Fig. 3. Début de la liste de lieux mnémoniques dans l'art de la mémoire écrit en 1431 à Erfurt par Mattheus Beran

La liste est organisée thématiquement, en incluant par exemple une section 'choses servant à écrire', 'choses servant à se réchauffer', 'à manger', etc. La plus grande partie de cette liste est extraite du traité composé en 1421 par Matthieu de Vérone. Prague, Bibliothèque nationale, I. F. 35, fol. 479r



Fig. 4. Innovation apportée par Mattheus Beran à la liste mnémonique de 100 places: on ne doit mémoriser qu'une sentence (alphabétique) de neuf mots («Abbas Bernardus cupit dare ecclesiam fratribus gratis hodie Ierosolimis»), puis ajouter à chaque dixième intervalle une couleur différente, en répétant dix fois l'opération.

(Ista ergo sunt 9 loca per numerum aphabeti descripta et secundum suum ordinem figurarum representativa. demum adorna decimum locum 10 albi, 20 viridem, 30 rubeum, 40 flaveum, 50 nigrum, 60 glaucum, 70 griseum, 80 ferreum, 90 argenteum, 100 aureum)

Prague, Bibliothèque nationale, I. F. 35, fol. 481r 


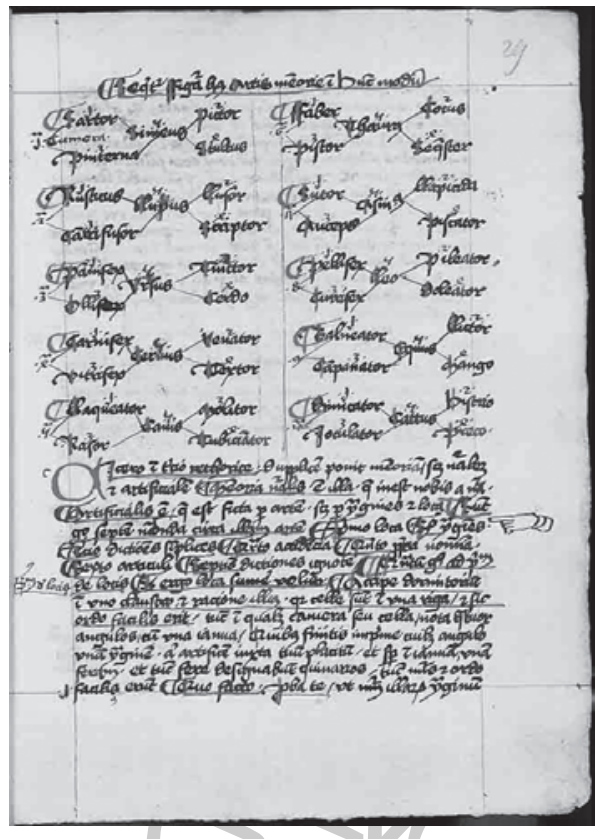

Fig. 5. Liste de lieux mnémoniques organisée visuellement en groupes de cinq, chacun d'entre eux consistant en un animal et quatre artisans

Prague, Bibliothèque nationale, I. G. 11a, fol. 29r (1491)

Dans les pays de la couronne de Bohême, on a conservé un extraordinaire fragment anonyme d'un traité provenant probablement des milieux hussites ${ }^{32}$. Les lieux de mémoire proposés dans ce traité embrassent par exemple les livres de Wycliff (libri wikleff), ou armée (exercitus). Le dernier groupe de cinq unités, en particulier, livre une image vivante de la violence apocalyptique:

Undecimus [quinarius]
Grex
Monachi comburuntur
Sepultura violantur
Vasa dirapiuntur
Terre fodiuntur

Undecimus [quinarius]

Monachi comburuntur

Sepultura violantur

Terre fodiuntur
onzième [groupe de cinq]:
troupeau
des moines sont brûlés
des sépultures sont pillées
des vases sont volés
des terres sont creusées

32 Inc. Nam secundum commentatores in libro de memoria et reminiscencia, à Prague, Bibliothèque nationale, VIII. E. 3 (écrit après 1415), fol. 136v-142r et 175v. Voir J. Truhlář, Catalogus codicum, t. I, Prague, 1905, n 1528, p. 561-562; P. Spunar, Repertorium auctorum Bohemorum provectum idearum post Universitatem Pragensem conditam illustrans, t. I (Studia Copernicana 25), Wrocław, 1985, nº 649. 


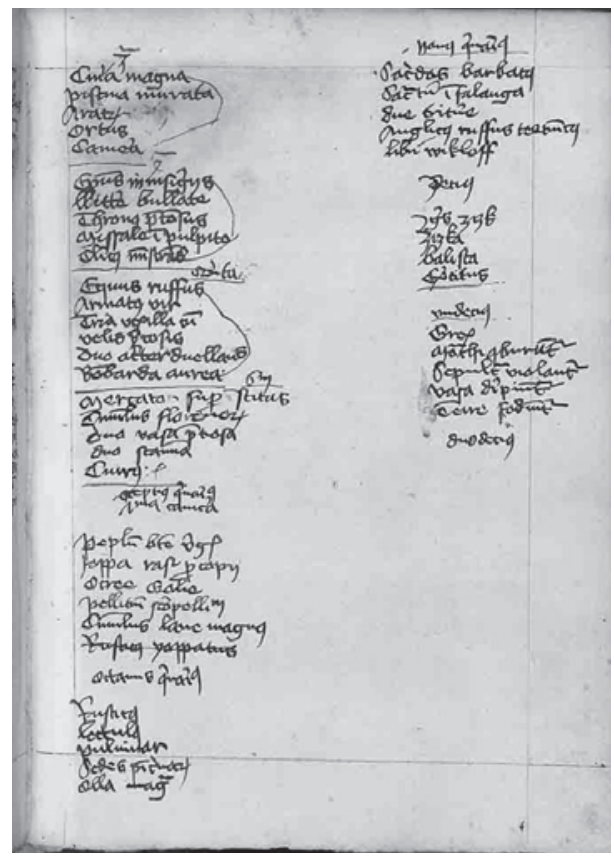

Fig. 6. Liste de lieux mnémoniques utilisée par un Hussite

Prague, Bibliothèque nationale, VIII. E. 3, fol. 142r (écrit après 1415)

Cet exemple illustre le caractère subjectif de l'art de la mémoire: cette structure particulière de cent places pourrait difficilement être considérée comme utile pour la plupart des étudiants médiévaux. En vue d'adhérer efficacement à une mémoire particulière, la structure en lieux a besoin d'être proche des intérêts de l'étudiant: elle doit être à la fois frappante et familière.

Ainsi, le catalogage alphabétique des mots, qui imprégnait la culture du Moyen Âge tardif à travers différents genres (chants ecclésiastiques, poèmes acrostiches, traités de méditation, sermonnaires, encyclopédies thématiques) agissait également comme une force de hiérarchisation importante dans l'art de la mémoire. Les termes possédant un pouvoir d'évocation mémorielle n'étaient néanmoins pas nécessairement agencés en fonction d'un ordre alphabétique; ils pouvaient également suivre des schémas géométricofiguratifs.

Un dessin d'Ulricus Crux de Telcz offre un exemple éclatant de la structuration figurative des mots dans un but mnémonique ${ }^{33}$. Ulricus Crux

33 F. G. Kiss, «Memory, Meditation and Preaching: A Fifteenth-Century Memory Machine in Central Europe (The Text Nota hanc figuram composuerant doctores... / Pro aliquali intelligen- 
de Telcz, ou en suivant la version tchèque de son nom, Oldřich Kř́íz Telče, un chanoine régulier de saint Augustin, a composé un dessin remarquable pour le cloître Sainte Dorothée à Vienne en Autriche en 1491, peut-être placé sur le mur du cloître. L'image, si elle a effectivement été réalisée, n'a pas survécu, mais nous en possédons une copie détaillée dans un manuscrit de la Bibliothèque Nationale de Prague, sous la cote I. G. 11a. Selon la note écrite à côté de l'image dans le manuscrit ${ }^{34}$, nous devons contempler cette figure, car elle nous influencera de diverses manières. Un autre manuscrit ${ }^{35}$, qui contient le même texte, sans image, fait allusion à deux peintures sur bois - malheureusement disparues - qui se trouvaient dans le couvent franciscain de Brünn et dans la Chartreuse de Königsfeld (Královo Pole), où la même image avait été reproduite pour favoriser la méditation (Planches 3 et 4).

Dans cette figure, les concepts-clés sont ordonnés de manière géométrique, comme nous le verrons. Pour mieux comprendre ses modalités d'emploi, il faut en examiner la tradition et celle de son texte explicatif, que nous appellerons dans ces lignes «Nota hanc figuram..." pour en faciliter la citation, car le traité est dépourvu d'incipit. Il commence par une image qu'explicite plus ou moins le texte. Quelques exemplaires (y compris le seul témoin imprimé, l'incunable de Nuremberg de 1473) transmettent à la fois l'image et le texte. Les titres qui se rencontrent dans ces manuscits sont Recordatio theologiae composita a doctoribus universitatis Parisiensis, Recordatio theologiae - Ludus paginae sacrae, ou Deus in se (ce qui correspond à l'inscription de la première image). Quelques autres exemplaires ne contiennent que l'image. Dans un seul manuscrit, nous trouvons l'image sous le titre Salvationis aeternae speculum, et il existe des xylographies colorées, qui ont été réalisées à partir de l'édition incunable ${ }^{36}$. Le troisième groupe - le plus nombreux - est celui des manuscrits qui ne contiennent que le texte explicatif. On a retrouvé jusqu'à présent quinze de ces manuscrits qui ne contiennent que le texte, et ont l'incipit Pro aliquali intelligentia praesentis figurae sciendum est... Le titre varie presque dans tous les cas: Flos artis praedicatoriae, Tractatus de modo praedicandi, De operibus Dei memorandis et praedicandis ou Recordatio theologiae.

tia...)», dans The Making of Memory in the Middle Ages, éd. L. Doležalová, Leiden, 2010, p. 4978 .

34 «Nota composicionem figure cum effectu aliquali 1491, descripta per me fratrem Crucem de Telcz sub abbate Marco de Trebon et priore Johanne de Straz, depicta Wienne in monasterio nostri ordinis sancte Dorothee.»

35 Olomouc, Vědecká knihovna, M I 156, 275b: Circuli 12 figure presentis picti sunt in quadam tabula lignea loci Brunensis et apud Carthusiam in Konigesfelt. Voir M. Boháček and F. Čáda, Beschreibung der mittelalterlichen Handschriften der Wissenschaftlichen Staatsbibliothek von Olmütz, Köln, 1994, p. 62. Le manuscrit a appartenu au couvent de Saint-Bernardin des Frères Mineurs à Brno (aujourd'hui église de Sainte-Marie-Madeleine): nous identifions le locus Brunensis avec le couvent franciscain de Brno.

36 Une copie se trouve dans la McGuire Collection de New York, une autre à Munich, où les feuillets xylographiques de l'édition sont reliés à un manuscrit. 
Les douze images sont rangées de deux manières: elles sont d'une part regroupées en fonction des six utilisations possibles des unités textuelles qui sont mémorisées à l'aide de cette superstructure (recordatio theologiae, paginae sacrae ludus, promptitudo praedicandi, artificium contemplandi, temptationes superandi, remuneratio futurorum). La signification exacte de ces termes n'est explicitée qu'à la fin du traité. En fait, ils se réfèrent au mode d'emploi des images et des textes: il est possible de se rappeler l'ensemble de la théologie grâce à leur aide (affirmation pour le moins audacieuse!), ou de jouer avec les Saintes Écritures (ludus paginae sacrae $)^{37}$. Le système peut également assister la prédication improvisée, ou la recherche de sujets adaptés à la contemplation.

L'autre division du cycle de douze images est organisée en fonction d'un schéma trois fois quatre. Les quatre premiers concepts, centraux, se rapportent à l'histoire du salut (Dieu, la Création, révocation, rédemption), le deuxième quatrain, à des circonstances de notre vie sur terre (la vertu, le vice, l'exil, le temps), tandis que les quatre dernières images correspondent aux «fins dernières » (mort, jugement dernier, paradis, enfer). Tout en ayant une notion centrale au milieu du champ, chaque image possède quatre autres notions subsidiaires, placées dans les coins. Ces notions subsidiaires sont en général étroitement liées au sujet de l'image centrale. Par exemple, Deitas, la Déité, qui est symbolisée par Sol (le Soleil) a comme concepts auxiliaires Potentia (puissance), Perfectio (perfection), Bonitas (bonté) et Sapientia (sagesse). Le traité, suivant les images, explique le contenu de ces coins à l'aide de citations. Chaque thème est clarifié par plusieurs citations de la Bible, des Pères de l'Église, ou d'autres autorités médiévales.

Le cas le plus éloquent d'un point de vue structurel est peut-être celui du Vice. Les trois premiers membres de la subdivision sont ici l'orgueil, l'avarice et la luxure (superbia, avaritia, luxuria) qui sont les trois premiers vices capitaux énumérés dans la liste condensée dans le mot mnémotechnique saligia. Un quatrième membre résume l'ensemble: c'est la deordinatio, qui, selon saint Thomas d'Aquin, est proprement la définition du péché (c'est à dire l'acte de subvertir l'ordre de Dieu, «déordination» $)^{38}$. Il faut naturellement penser à ces quatre sujets avec répulsion et horreur. La nécessité de les détester tous les quatre est prouvée par quatre citations pour chacun d'entre eux: par exemple, dans le cas de l'avarice, l'auteur anonyme cite deux fois les Épîtres pauliniennes, une fois la Sagesse de Salomon, une fois l'Évangile de Matthieu.

On peut aussi reconnaître d'autres logiques structurantes. Une série de sujets méditatifs débute avec la divinité ou le Soleil, qui est identifié avec la

37 On connaît la production de jeux mnémotechniques au début du xvi ${ }^{\mathrm{e}}$ siècle (le Chartiludium logicae de Thomas Murner, 1507/9, ou la Grammatica figurata de Matthias Ringmann, 1509). Ce traité, cependant, leur est antérieur.

38 Cfr Thomas d'Aquin, S. Th. 1a 2ae, q. 72, a. 5. 
puissance (potentia) dans l'en-tête de sa description. La Création, second membre de la série, est dérivée de la perfection de Dieu, selon le titre du chapitre ("parfaite, car elle brille dans sa création et dans son ordre parfait»), et les troisième et quatrième membres (revocatio - le rappel de celui qui est égaré, et redemptio - la rédemption de l'humanité) sont dérivés de la troisième qualité de la Déité (ou Soleil), c'est-à-dire la bonté. Les cinquième et sixième membres, le Vice et la Vertu, découlent logiquement de l'objet des troisième et quatrième membres (la révocation et la rédemption des péchés), et les divisions de la Vertu reflètent parfaitement celles du Vice: l'orgueil est contrebalancé par l'humilité, l'avarice par la générosité, la luxure par la chasteté, et la «déordination » par l'ordination, l'obéissance à l'ordre divin. Le septième membre de la série, l'exil, est relié aux deux précédents par le premier article (ou coin) du Vice: d'après l'auteur anonyme, pour éviter de tomber dans le péché d'orgueil, il faut toujours méditer sur l'exil que représente notre vie mondaine, et la mention d'Adam lui donne l'occasion d'insérer le temps, Tempus, qui contient la division de l'histoire humaine dans les trois périodes successives de la lex naturae (jusqu'à Moïse), la lex scripturae (jusqu'à Jésus) et la lex gratiae. Enfin, le thème du libre arbitre leur est rattaché, afin de parachever la structure quadripartite.

Jusqu'à présent, la structure du traité a suivi une ligne logique, même si cette logique était manifestement improvisée. Les quatre derniers sujets, en revanche, font partie d'une tradition littéraire qui existait déjà depuis la fin du XIV" siècle, la doctrine des "quatre fins dernières". Selon la logique explicite du traité, il faut méditer sur ces sujets, parce que le temps nous offre une chance d'utiliser notre libre-arbitre, donc le temps est logiquement suivi par la mort et le Jugement dernier, après lesquels nous attend soit le paradis, soit l'enfer, en fonction de nos mérites.

L'ordonnancement alphabétique des termes peut ainsi être remplacé par une structure mi-logique, mi-figurative. Dans le cas du traité Nota hanc figuram, les concepts-clés apparaissant dans l'image mnémonique s'organisent en une séquence qui conduit de Dieu et de la création jusqu'au salut et au destin de l'homme. Le schéma des douze images autour duquel le traité est structuré est clairement mnémonique: il apparaît pour la première fois dans un traité sur l'art de la mémoire, écrit à Bologne en $1425^{39}$. Ce traité remplit toutefois les espaces vides qui sont laissés en blanc dans les artes memorativae pour y placer le matériel à mémoriser, et investit ses mots-clés du pouvoir de faire surgir dans la mémoire un répertoire mental entier de citations d'autorités, concernant un large spectre de sujets théologiques.

39 Voir R. A. Pack, «An Ars memorativa from the Late Middle Ages», Archives d'histoire doctrinale et littéraire du Moyen Âge, 36 (1979), p. 265-266 et F. G. Kiss, « Performing from Memory and Experiencing the Senses in Late Meditative Practice », Daphnis, 41 (2012), p. 419-452. 


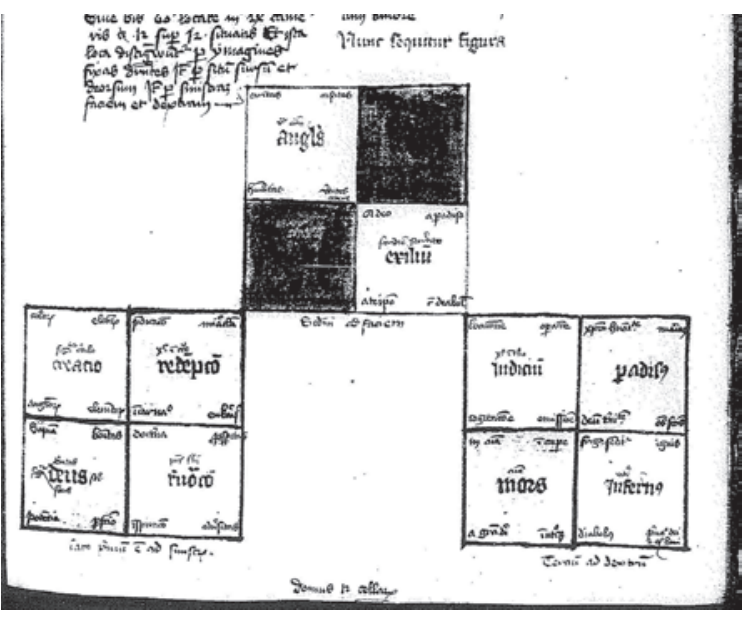

Fig. 7. Dessin pour la méditation dans l'art de la mémoire Memoria fecunda Wien, Österreichische Nationalbibliothek, cod. 4444, fol. 327r (1425)

\section{IMAGES DE LA MÉMOIRE: LE CORPS ET L'ESPRIT DES MOTS}

Les images mémorielles fonctionnent sur la base de la transformation: la chose qui doit être remémorée est manipulée, changée en quelque chose d'autre, sur la base d'une ressemblance quelconque. Plusieurs modes consistant à imposer artificiellement une signification sont utilisés dans la création des imagines au sein de l'art de la mémoire. En général, l'association lexicale et la symbolique visuelle jouent un rôle essentiel lors de la création des images. On constate que les deux aspects sont déjà présents et associés dans la description de l'image complexe que propose l'Ad Herennium:

Rei totius memoriam saepe una nota et imagine simplici conprehendimus; hoc modo, ut si accusator dixerit ab reo hominem veneno necatum, et hereditatis causa factum arguerit, et eius rei multos dixerit testes et conscios esse: si hoc primum, ut ad defendendum nobis expeditum 〈sit, > meminisse volemus, in primo loco rei totius imaginem conformabimus: aegrotum in lecto cubantem faciemus ipsum illum, de quo agetur, si formam eius detinebimus; si eum non, at aliquem aegrotum «non > de minimo loco sumemus, ut cito in mentem venire possit. Et reum ad lectum eius adstituemus, dextera poculum, sinistra tabulas, medico testiculos arietinos tenentem: hoc modo et testium et hereditatis et veneno necati memoriam habere poterimus. Item deinceps cetera crimina ex ordine in locis ponemus; et, quotienscumque rem meminisse volemus, si formarum dispositione et imaginum diligenti notatione utemur, facile ea, quae volemus, memoria consequemur.

Souvent un signe unique, une seule image suffisent à nous assurer le souvenir de tout un événement. Par exemple, l'accusateur prétend que le prévenu a empoisonné un homme, l'accuse d'avoir commis le crime pour s'assurer un 
héritage, et dit qu'il y a, pour le prouver, beaucoup de témoins, beaucoup de gens ayant été dans la confidence. Si nous voulons nous rappeler ce premier point, afin de pouvoir facilement présenter la défense, dans une première case nous nous tracerons une représentation de toute l'affaire. Nous nous représenterons étendu dans son lit, malade, l'homme même dont il est question, si nous connaissons ses traits; à son défaut, une personne quelconque, mais n'appartenant pas aux dernières classes de la société, afin que l'autre puisse vite nous revenir à l'esprit. Et, debout près de lui, à côté de lui, nous placerons l'accusé, tenant de la main droite le poison, de la main gauche des tablettes et des testicules ordinaires de bélier, par ce moyen nous pourrons nous souvenir des témoins, de l'héritage et de l'homme empoisonné ${ }^{40}$.

Ainsi est-il par exemple possible de mémoriser les témoins (testes) par l'intermédiaire des testicules (testes), image incapable à elle seule de visualiser les témoins dans notre mémoire. Il faut donner son nom à cette image pour trouver la clé de l'énigme.

Bien que les aspects verbal et visuel de la pratique de création des images soient interconnectés et difficilement séparables, il est clair que les mots opèrent également comme des déclencheurs/incitateurs de mémoire. Pour acquérir leur efficacité dans l'art, les mots sont manipulés de deux manières simples: c'est soit leur forme, soit leur contenu qui est transformé, c'est-à-dire que le changement a lieu soit au niveau des lettres et des syllabes, soit au niveau sémantique.

\section{LE CORPS : LE NIVEAU FORMEL}

Le niveau formel a été présenté au début de cet article: les mots sont coupés, l'ordre de leurs lettres ou de leurs syllabes altéré, et certaines lettres ou syllabes peuvent même être remplacées par d'autres. Le mot résultant ressemble à l'original, tant en ce qui concerne les lettres que le son. Le lien n'est pas sémantique, mais formel. Le lecteur doit réorganiser les lettres et/ ou les syllabes pour arriver à la solution. C'est une sorte d'énigme, un moyen spécifique de retarder l'accès à la signification.

$\mathrm{Ce}$ «sciage des mots » était une stratégie mnémotechnique très populaire à la fin du Moyen Âge, mais il ne se restreint ni à l'art de la mémoire, ni au Moyen Âge tardif. Au viI ${ }^{\mathrm{e}}$ siècle déjà, le grammairien intrigant répondant au nom de Virgilius Maro Grammaticus ${ }^{41}$ parle du «sciage» des mots, scinderationes fonorum, et de son emploi dans trois buts possibles:

40 Ad Her III, 20.

41 On sait peu de choses sur lui. Bien qu'il n'y ait aucun témoignage direct, son origine irlandaise a été avancée à plusieurs reprises par M. Herren ("Some new light on the life of Virgilius Maro Grammaticus", Proceedings of the Royal Irish Academy 79, section C (1979), p. 2771), et ses travaux sont souvent considérés comme des précurseurs de la tradition poétique 
$\mathrm{Ob}$ tres causas fona finduntur : prima est ut sagacitatem discentium nostrorum in inquirendis atque in inveniendis his quaeque obscura sunt adprobemus. Secunda est propter decorem aedificationemque eloquentiae. Tertia ne mystica quaeque et quae solis gnaris pandi debent passim ab infimis ac stultis facile repperiantur, ne secundum antiquum sues gemmas calcent.

(...primo, pour assurer l'acuité de la perception de nos étudiants lors de la recherche et de la trouvaille de ces choses obscures; secundo, en raison de l'ornement et de la construction du discours; tertio, pour que les secrets mystiques, qui doivent être connus des seuls savants, ne soient pas par hasard découverts par des êtres inférieurs et stupides, pour que les perles ne soient pas ainsi jetées devant les pourceaux ${ }^{42}$.)

On ne parle d'habitude que des deux dernières utilisations : l'emploi des jeux de mots et des devinettes d'une part, en tant que figures rhétoriques donnant davantage d'attrait formel au texte; l'encodage des informations secrètes d'autre part. C'est d'ailleurs ainsi que l'on a analysé jusqu'à maintenant l'œuvre de Virgilius Maro Grammaticus lui-même, les chercheurs restant persuadés soit qu'il jouait d'une manière absurde, soit au contraire, que son texte cachait des secrets mystiques. Mais c'est précisément la troisième raison d'utiliser cette technique qui est au Moyen Âge la plus répandue, et ce à divers niveaux. Présente dans l'art de la mémoire, elle se retrouve également dans la mnémotechnique et dans la pratique médiévale de l'écriture.

Dans le contexte de l'art de la mémoire, un exemple plus détaillé nous est fourni par un traité déjà évoqué, l'Ars memorativa de Mattheus Beran, moine au couvent augustinien de Roudnice. Après la destruction de ce couvent par les Hussites en 1421, il fut actif à Erfurt, puis à Bâle. C'est, semblet-il, à Erfurt qu'il écrivit son Ars memorativa ${ }^{43}$. Le traité semble n'être conservé que dans un seul manuscrit complet, qui est également autographe ${ }^{44}$. Il est

irlandaise spécifique plus tard développée dans les Hisperica famina, avant de culminer dans le Finnegan's Wake de James Joyce. Vivien Law pensait que cet auteur était peut-être anglais (V. Law, Insular Latin Grammarians, Woodbridge, 1982, p. 87). Virgilius lui-même mentionne de manière indirecte son origine galloise. Abbon de Fleury l'appelle Tolosanus (toulousain). On l'a également considéré comme un juif: plusieurs des mots étranges qu'il utilise semblent d'origine hébraïque. L'idée la plus curieuse (et la moins acceptée) est celle de Leo Wiener, qui voit en Virgilius un auteur issu de la culture arabe, et interprète la plupart de ses obscurités comme des termes d'origine arabe, v. L. Wiener, Contributions Toward a History of Arabico-Gothic Culture, Piscataway, 2002, p. 21.

42 Epitome 10. Édition la plus récente par Bengt Löfstedt, Virgilius Maro Grammaticus: Opera Omnia, Munich, 2003. Les éditions précédentes sont de G. Polara (trad.), L. Caruso et G. Polara (éd.), Virgilius Maro Grammaticus, Epitomi ed Epistole, Nuovo medioevo 9, Napoli, 1979, p. 128 ; ainsi que de J. Huemer, ed., Virgilii Maronis grammatici opera, Leipzig, 1886, p. 76.

43 Voir L. Doležalová, «Fugere artem memorativam? The Art of Memory in 15th c. Bohemia and Moravia (A Preliminary Survey)», Studia mediaevalia Bohemica, 2:2 (2010), p. 234-241.

44 Prague, Bibliothèque nationale, I. F. 35, achevé le 12 mai, 1431 (cfr fol. 485r : «...per me fratrem M. Beran exulem canonicum regularium de Rudnicz manu mea propria... anno domini 1431 sabbato post ascensionem domini in Erfordia in domo pauperum»): voir J. Truh- 
organisé de manière assez confuse, avec une double introduction. Mais les «images» proposées par Beran sont des exemples appropriés dans la mesure où les mots qui en sont le support se prêtent à une transformation lexicale au service de la mémorisation. En fait, il s'agit d'une sorte de suite de jeux de mots. Nous devrions par exemple, selon Beran, mémoriser Thomas d'Aquin comme un homme avec un gouvernail, themo navis. Le gouvernail, themo, doit apparemment rappeler Thomas, Thoma, et le navire l'eau, aqua, grâce à quoi nous devrions nous souvenir de Aquin, Aquinas. Saint Ambroise doit en revanche porter au cou un chapelet d'ambre (Ambrosius - ambre $)^{45}$. Parfois, la même image peut servir à mémoriser deux choses différentes: ainsi le genu ferreum (genou de fer) devrait correspondre à la fois au livre de la Genèse et au génitif singulier.

L'analyse révèle que Beran a en fait copié la plus grande partie de son traité sur l'œuvre d'un autre Mattheus, Matthieu de Vérone, une figure bien documentée ${ }^{46}$ dont le traité sur la mémoire, rédigé en 1420 et remanié en 1423, est conservé dans au moins neuf manuscrits ${ }^{47}$. Le travail de Beran est une copie au sens médiéval du terme - il abrège, saute, complète, remanie, etc. Grâce à ce cas particulier, il est possible de discerner l'importance, pour l'art de la mémoire, de la langue vernaculaire dissimulée à l'arrière-plan des formes latines. Certaines des images que Matthieu de Beran suggère restent en effet obscures sans la consultation des propositions originales de Matthieu de Vérone. Par exemple, alors que dans le texte de Beran, probablement corrompu à cet endroit, l'image d'Origène est associée à celle d'un homme portant au cou un instrument ([imago] Origenis unus cum portatico ad collum), cette formule s'éclaire en lisant Matthieu de Vérone, qui écrit: unus cum uno organo paruo (un homme avec un petit orgue portatif, ou tout autre instrument de musique) - version qui implique une vraie ressemblance avec le nom de l'écrivain (Origenes/organum). Matthieu de Vérone orthographiait également le couvre-chef associé à saint Bernard berretum (orthographe commune en Italie) plutôt que birretum comme Beran: la première version rappelle plus clairement Bernardus.

Néanmoins, le traité de Beran est une copie: Matthieu Beran a même copié l'incipit en remplaçant de Verona par Beran. Il semble avoir découvert quelque part le traité de Matthieu de Vérone. Peut-être ébloui par la

lář, Catalogus codicum manu scriptorum latinorum qui in C. R. Bibliotheca publica atque universitatis Pragensis asservantur, t. I, Prague, 1905, p. 110-111, no. 267. Dans la seconde moitié du Xve siècle, Oldřich Kř́̌ž de Telč a recopié un fragment de ce traité dans Prague, Bibliothèque nationale, I. G. 11a, fol. 29v-30r.

45 «Sit ymago sancti Thome de Aquino habens themonem navis ad collum, sancti Ambrosii unus habens cordam ad collum cum pater noster de ambra " (Prague, Bibliothèque nationale, I. F. 35, fol. 482r).

46 Voir Seelbach, Ars und Scientia, p. 34-38.

47 Voir Seelbach, Ars und Scientia, p. 35. Le traité n'étant pas encore édité, il est impossible de comparer ces textes en détail. 
ressemblance de son nom avec celui de l'auteur, il a profité du jeu de mots, c'est-à-dire de la stratégie dont il avait eu connaissance précisément grâce à l'art de mémoire. Il a ainsi créé une «image» de même nature que celles contenues dans le traité. Ce faisant, il ne tentait pas de la proposer comme un outil mémoriel, mais plutôt de se dissimuler! Cette stratégie reflète l'emploi pratique du «sciage » et de la transformation des mots, utilisée à la fois pour des fins de mémorisation et dans d'autres buts.

Cette méthode de «sciage» des mots est omniprésente dans la littérature médiévale: on trouve souvent des «mots brisés» hors du contexte de l'art de mémoire. En vertu de sa culture manuscrite, l'expérience des «mots brisés» était quotidienne pour le lecteur médiéval. Il avait l'habitude de corriger, rétablir et recomposer ces mots dans son esprit, découvrant ainsi des possibilités qui s'étendaient au-delà même de ces mots et de leur signification initiale ${ }^{48}$.

\section{L'ESPRIT : LE NIVEAU SÉMANTIQUE}

Dans la plupart des cas, toutefois, le corps des mots est laissé intact, et ce sont les pouvoirs associatifs ou subversifs cachés dans leur signification première qui sont utilisés pour créer des relations mnémoniques. L'art de la mémoire envisage les mots du langage de sorte que n'importe quel mot ou phrase, même les plus innocents ou les moins ambigus, peut recevoir un nouveau sens, et l'effort mental investi dans la création de ces nouveaux liens peut être utilisé comme un outil de mémorisation. Même les noms propres, qui par définition ne devraient avoir qu'un seul référent, peuvent être investis de ce pouvoir en étant placés dans un nouveau contexte, un environnement neuf, qui peut être ridicule ou terrifiant, afin de satisfaire aux exigences de la mémorisation.

Nombre de traités mnémoniques suggèrent ainsi qu'une citation de saint Antoine ou saint Thomas devrait être mémorisée grâce à un Antoine ou Thomas spécifique, que l'on connaît personnellement, simplement en plaçant le porteur de ces prénoms dans une situation burlesque. Giovanni Michele Alberto Carrara, humaniste italien de la seconde moitié du $\mathrm{xv}^{\mathrm{e}}$ siècle originaire de Bergame, indique en citant Avicenne qu'il faut convoquer l'image de belles jeunes filles dont les noms commencent par la même lettre que celle de la chose dont on doit se souvenir. Il choisit pour présenter la construction des images drôles ou émouvantes l'exemple suivant: si nous voulons mémoriser une chose liée à Antoine, nous devons imaginer l'un de nos amis, dont le nom est Antoine, placé dans une situation où sa tête est mordue par un âne enragé,

48 Voir L. Doležalová, «On Mistake and Meaning: Scinderationes fonorum in Medieval Artes Memoriae, Mnemonic Verses, and Manuscripts ", Language and History, 52 (2009), p. 25-39. 
où son sang coule et où il appelle désespérement à l'aide ${ }^{49}$. Pierre de Ravenne, professeur de droit et célèbre enseignant de l'art de la mémoire vers 1500, confessait non sans honte qu'il utilisait les images de superbes jeunes filles comme lettres ${ }^{50}$. Christian Umhauser, auteur d'un traité allemand écrit vers 1500 , suggère que pour mémoriser une potion, nous imaginions un docteur renommé, drapé dans de superbes habits, qui tient une fiole d'urine à la main, et la renverse sur une vieille femme. Haec est pulchra imago, dit l'auteur ${ }^{51}$. En règle générale, tout mot ou action peut être subverti pour obtenir un effet ridicule ou horrible: «Tunc ymaginem cuiuslibet dictionis pone ad locum sive ad differentiatorem loci cum sua actione ridiculosa, et hoc est valde facile et maxime utilitatis, ut bene patebit practicanti ${ }^{52}$ ».

Néanmoins, le plus souvent, ce n'est pas simplement la recontextualisation d'un mot, mais également son pouvoir d'association qui redéfinit sa signification mémorielle. La création de listes lexicales dotées de tels sens «secondaires » était l'une des méthodes les plus communes pour produire un vocabulaire mnémonique dans lequel chaque mot signifiait quelque chose d'autre que son sens premier. Le lecteur moderne peut comprendre aisément la raison justifiant certaines relations d'association, alors que d'autres restent énigmatiques, parce que ces liens peuvent avoir existé seulement pour l'individu qui y recourt en fonction de sa mémoire individuelle ou de ses

49 G. M. A. Carrara, De omnibus ingeniis augendae memoriae, Bologna, Plato de Benedictis, 1491, fol. a5r: «ut risum moveat figura, aut misericordiam aut admirationem, haec enim facit etiam puellas recordari, ut inquit Avicenna sexto naturalium particula quarta. facile enim inuenitur quesita figura que affectum anime commouerit: exemplum hoc est. in ore asini rabidi caput Antonii constituam morsibus fere ossa confringi. cruorem effluere illum auxilia petere, et passis palmis vociferare. fieri non poterit, ut cum uoluero non uideam hunc oculis mentis meae, et reddere Antonium nesciam repetenti.»

50 «...et ego communiter pro literis formosissimas puellas pono: illae enim multum memoriam meam excitant. [...] et mihi crede, si pro imaginibus pulcherrimas puellas posuero, facilius et pulchrius recito que locis mandavi [...] quod diu tacui ex pudore», dans Petrus Ravennas, Foenix, Venise, Bernardinus de Choris, 1491, b4v. Il est difficile de savoir s'il pense à des jeunes filles dont les noms commencent par les mêmes lettres que les choses qu'il doit mémoriser, ou à un alphabet figuratif constitué par de belles filles.

51 «Imago (ut antea dixi) est similitudo et figura et significatio rei, quam volumus locis tradere. Verbi gratia, si vellem commemorare medicinam, ad locum constituo medicum mihi cognitum mirabili veste indutum urinale in manu habens et urina vetulam respergens. Hec est pulchra imago. In ordine regula: Imagines debent esse rarae, mirabiles, inusitatae, ridiculae, quia natura usitata re non exsuscitatur et debemus eis attribuere egregiam pulchritudinem aut unicam turpitudinem si aliquas exornabimus aut corona aut veste, tunc cruentam aut steno oblitas inducamus »; Munich, Bayerische Staatsbibliothek, Clm 4417, 3r-v. Cette citation n'apparaît pas dans la version imprimée du texte, C. Umhauser, Ars memoratiua S. Thome, Ciceronis, Quintiliani, Petri Rauenne, Nürnberg, 1501.

52 [Magister Hainricus], «Ars memorativa», dans Ars vitae contemplativae, Modus meditandi in generali, Ars memorativa, Pseudo Thomas de Aquino: Ars praedicandi, Modus formandi arborem, [Nürnberg, Friedrich Creussner], 1473, 13r. Sur cet auteur, v. Seelbach, Ars und Scientia, p. 5054, 254-269. 
perceptions propres. Michele del Giogante étudia l'art de la mémoire sous la direction de Niccolò Cieco d'Arezzo à Florence en 1435. La méthode de maître Niccolò était de créer une liste de cent items à partir d'objets contenus dans la demeure de l'étudiant, pour placer ensuite « un objet par dessus chacun d'eux» (quello è posto sopra a questi luoghi per arme), c'est à dire de demander à Michele ses associations personnelles avec lesdits objets. Ainsi l'implication personnelle, le travail mental investis dans la création de ces associations assuraient-ils leur fixation dans la mémoire. Michele avait très souvent recours à ces significations codifiées qui étaient très diffusées en raison de leur place dans le symbolisme et l'iconographie chrétienne, quoiqu'elles n'aient probablement guère de valeur pratique dans la vie quotidienne. Par exemple, une colonne devant la maison devenait un signe mnémonique pour Samson aveuglé (La colonna pure in quella faccia: Sansone ciecho, 346-347). Il employait également des associations à deux niveaux: le puits de la maison était supposé être le puits de la Samaritaine, qui offrait donc un marqueur mnémonique pour Jésus (Il pozzo della Samaritana cioè il pozzo nostro: Giesu di sopra, 346-347), un tonneau de vinaigre (probablement associé aux réserves de vinaigre de la maison) signifiait un Juif (340-341: Botte dell'acieto, da man ritta della volta: Un giudeo di sopra). Dans ces cas, le pouvoir d'association des mots «puits » et "vinaigre» est assez clair, à cause du puits de la Samaritaine (Jn 4, 4-26), et du vinaigre offert deux fois au Christ lors de sa crucifixion (Mt 27, 32-36, Jn 19, 23-30).

Une semblable liste d'associations personnelles survit dans le manuscrit de Valentinus de Monteviridi, chanoine de Vác (Waitzen), qui avait recopié un art de la mémoire en Hongrie en 1504, d'après le colophon de son texte ${ }^{53}$. Ce texte est une variante réécrite d'un traité qui est attribué par la tradition à Conrad Celtis ${ }^{54}$. D'après cet art de la mémoire, il faut mémoriser un palais alphabétique de vingt pièces, chacune d'entre elle contenant cinq mots, ou images d'autres mots, qui agissent comme pivots du système. Chacun de ces 100 éléments doit être associé individuellement avec quelque chose dont cette image sera dorénavant le signe. Valentinus avait lié ses propres associations à chacun des mots de l'alphabet mnémonique de Celtis, jusqu'à la lettre $M$, et il avait même changé la signification de sept éléments qui étaient clairement définis par Celtis. Un abbé signifiait la religion pour Celtis, la chasteté (castitas)

53 Wrocław, Ossolineum, 734/I, fol. 168r-171r (1504 Wacie in profesto trinitatis). Sur ce texte, voir F. G. Kiss, «Valentinus de Monteviridi (Grünberg) ...», cit. supra n. 28.

54 Ce traité a été imprimé pour la première fois en 1492 dans l'Epitoma in utramque Ciceronis rhetoricam de Celtis, mais comme les deux autres sections de ce livre, un abrégé rhétorique et un traité d'art épistolaire, n'ont pas été écrits par lui, mais probablement rédigés à partir de notes prises durant ses leçons, il nous semble probable que les traités de Valentinus et Celtis remontent à un ancêtre commun. Voir Fr. J. Worstbrock, Die 'Ars versificandi et carminum' des Konrad Celtis, Ein Lehrbuch eines deutschen Humanisten, = Studien zum städtischen Bildungswesen des späten Mittelalters und der frühen Neuzeit, éd. B. Moeller, H. Patze, et K. Stackmann, Göttingen, 1983, p. 462-498, ici p. 470-474. 
pour lui, alors que la religion était signifiée par un cardinal (cardinalis); un chevalier (eques) signifiait la justice pour Celtis, mais le brigandage (rapacitas) pour Valentinus, car c'était l'empereur (Cesar) qui maintenait la justice. L'administrateur (officialis) rappelle chez lui la citation en justice (citatio), au lieu du procès. Le baigneur devient une personne au teint hâve (pallidus), alors que chez Celtis il signifiait des individus sales. La béguine enfin, qui signifiait la superstition chez l'humaniste allemand, est ici associée à la querelle (rixe). Il a consigné les associations qu'il inventait pour chaque mot, et les a répétées en petits caractères au-dessus des mots eux-mêmes, ce qui suggère qu'il a tenté de les mémoriser en pratique. C'est une vue partiale du monde qui se déploie à travers ces associations, où la musique apporte la joie (musice = iocunditas), les cuisiniers utilisent le gingembre (cocus = sisiber), les goûteurs

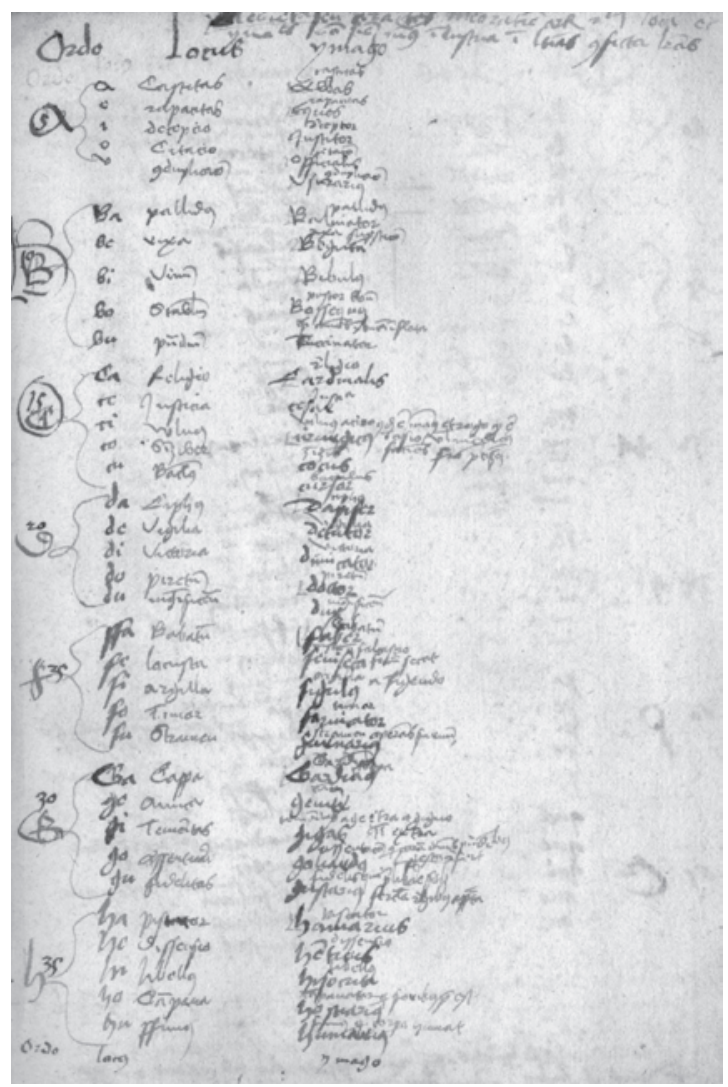

Fig. 8. Valentinus de Monteviridi, Notes mnémoniques Wrocław, Ossolineum, 734/I, fol. 170r (1504). 
professionnels doivent être fidèles (gustator $=$ fidelis), les poètes n'ont que louange à la bouche (goliardus = assentacio), et l'usurier exige toujours le double de son prêt (usurarius = conduplicacio).

Tout comme dans le cas de Michele del Giogante, toutefois, nous sommes incapables d'expliquer pourquoi certains mots ont été associés à d'autres termes par Valentinus de Monteviridi. Nous pouvons imaginer pourquoi il reliait la peur à l'image d'un adultère (fornicator $=$ timor), ou une petite amie à une mère (amica $=$ genetrix), mais la raison cachée de ces associations reste toujours personnelle.

En définitive, la création artificielle ou la recréation de liens entre des mots et des idées imprégnait la pensée du Moyen Âge tardif, et se retrouvait dans une multiplicité de formes. Les mots pouvaient être brisés, jusque dans leurs lettres et syllabes, afin de renouveler la signification qui était la leur dans la mémoire humaine. Des mots entiers pouvaient être utilisés comme des symboles d'association avec quelque chose d'autre que leur sens originaire. Des édifices plus importants, construits à partir de mots pour former des diagrammes de plusieurs unités lexicales, devaient également servir à mémoriser des structures abstraites, requises comme outils rhétoriques ou méditatifs afin d'indiquer l'ordre d'argumentation à suivre dans les prises de parole publiques, ou afin d'aider la méditation pour obtenir le salut de l'âme. Comme nous l'avons vu, ces outils acquirent une popularité sans précédent au cours du $\mathrm{Xv}^{\mathrm{e}}$ siècle, un phénomène qui reste difficilement explicable, même si la diffusion de l'éducation universitaire, les méthodes de prédication des ordres mendiants et l'émergence du livre imprimé peuvent être considérés comme des facteurs déclenchants de premier ordre dans ces changements.

Le facteur qui contribua peut-être de la manière la plus importante à accroître la flexibilité des structures sémantiques fut l'importance de l'allégorie, ou plutôt, en se servant d'un terme proposé par Gilbert Dahan, du "saut herméneutique» dans la pensée médiévale ${ }^{55}$. Thomas d'Aquin, après Augustin, Bède et tant d'autres, mentionna souvent les processus sémantiques de double signification, signification d'une chose à partir d'un mot, signification d'une chose à partir d'une autre chose, dans ses réflexions sur l'exégèse:

Dieu peut écrire avec des choses, pas seulement avec des mots... En effet, d'après ce qui a été dit, ces sens ne se multiplient pas pour cette raison qu'un seul mot signifierait plusieurs choses, mais parce que les réalités elles-mêmes,

55 Cfr G. Dahan, L'exégèse chrétienne de la Bible en Occident médiéval, XII -XIV siècles, Paris, 1999, p. 299, 441-445 et Id., Lire la Bible au Moyen Âge: Essais d'herméneutique médiévale, Genève, 2009 , p. 233-242. 
signifiées par les mots, peuvent être signes d'autres réalités (Summa theologiae, Ia, q. 1., art. 10. resp.).

Ainsi, avec l'aide des lectures figuratives, la connexion entre deux réalités peut être découverte, et le sens parabolique n'a pas une valeur moindre que le sens littéral.

Le sens parabolique est inclus dans le sens littéral; car par les mots on peut signifier quelque chose au sens propre, et quelque chose au sens figuré; et, dans ce cas, le sens littéral ne désigne pas la figure elle-même, mais ce qu'elle représente (Summa theologiae, Ia, q. 1., art. 10. resp. 3.).

L'imposition artificielle d'un sens aux mots et aux objets dans l'art de la mémoire est par bien des aspects similaire aux différents types de signification répertoriés à la fois dans l'exégèse et dans les théories sémantiques médiévales.

Comme dans le cas de l'allégorie, il reste difficile d'estimer à quel point de telles impositions sont arbitraires ou, au contraire, fondées sur une base solide. Pouvons-nous trouver des limites et des règles susceptibles de rendre compte de la création de telles significations? Il est rare de pouvoir observer ce système en action, car par définition, l'art de la mémoire est mental et oral, non écrit. Mais à partir d'exemples comme celui du traité hussite, avec ses éléments tchèques caractéristiques, ou celui de la maison mémorielle de Michele del Giogante, ou encore les associations de Valentinus de Monteviridi, qui permettent de voir le pouvoir des mots en action dans l'art de la mémoire tardo-médiéval, il devient possible de déduire qu'il existait des règles pour créer ces liens entre les mots, et de voir des schèmes de pensée émerger de ces associations à première vue dénuées de sens et dépourvues de convention. 


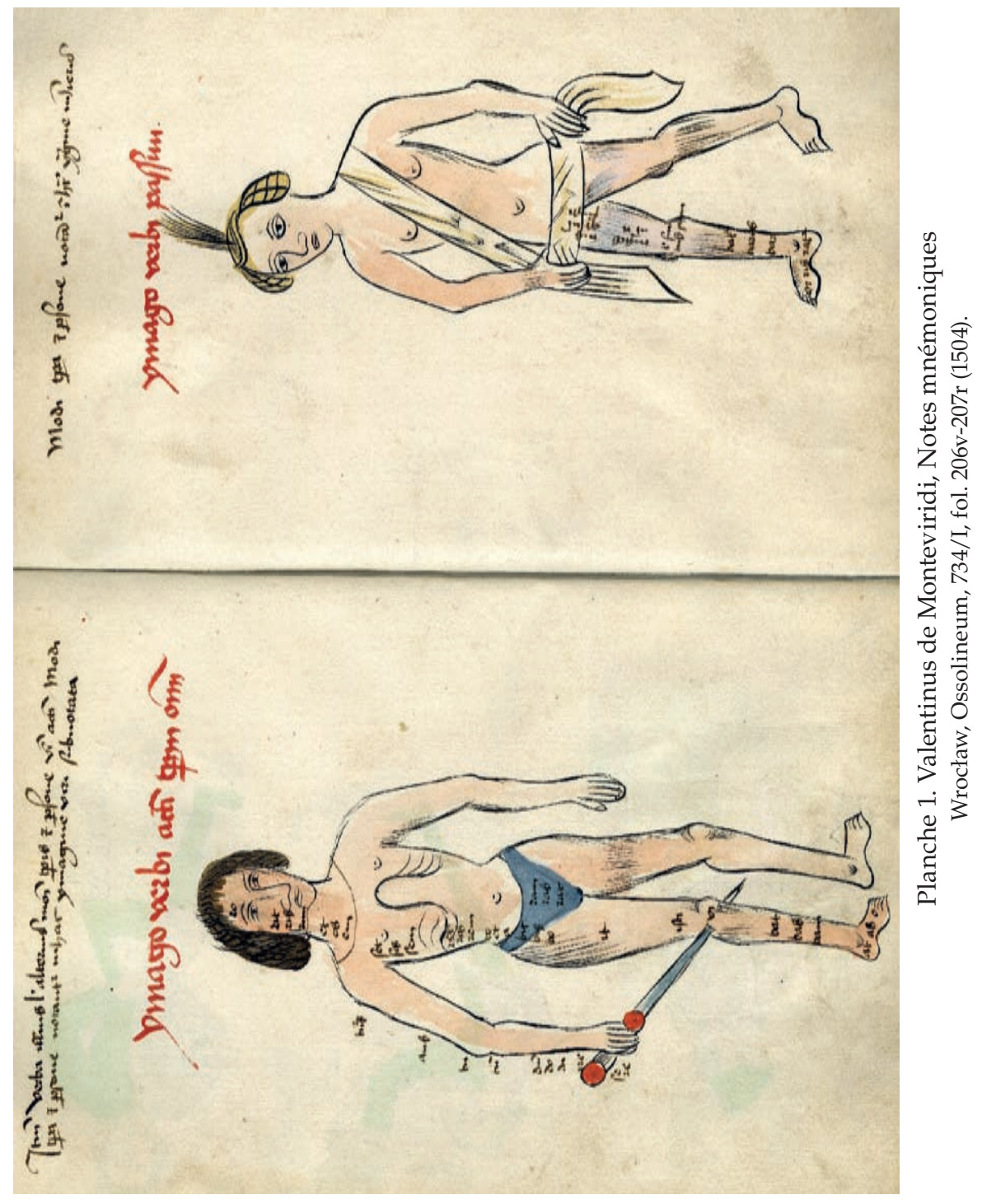




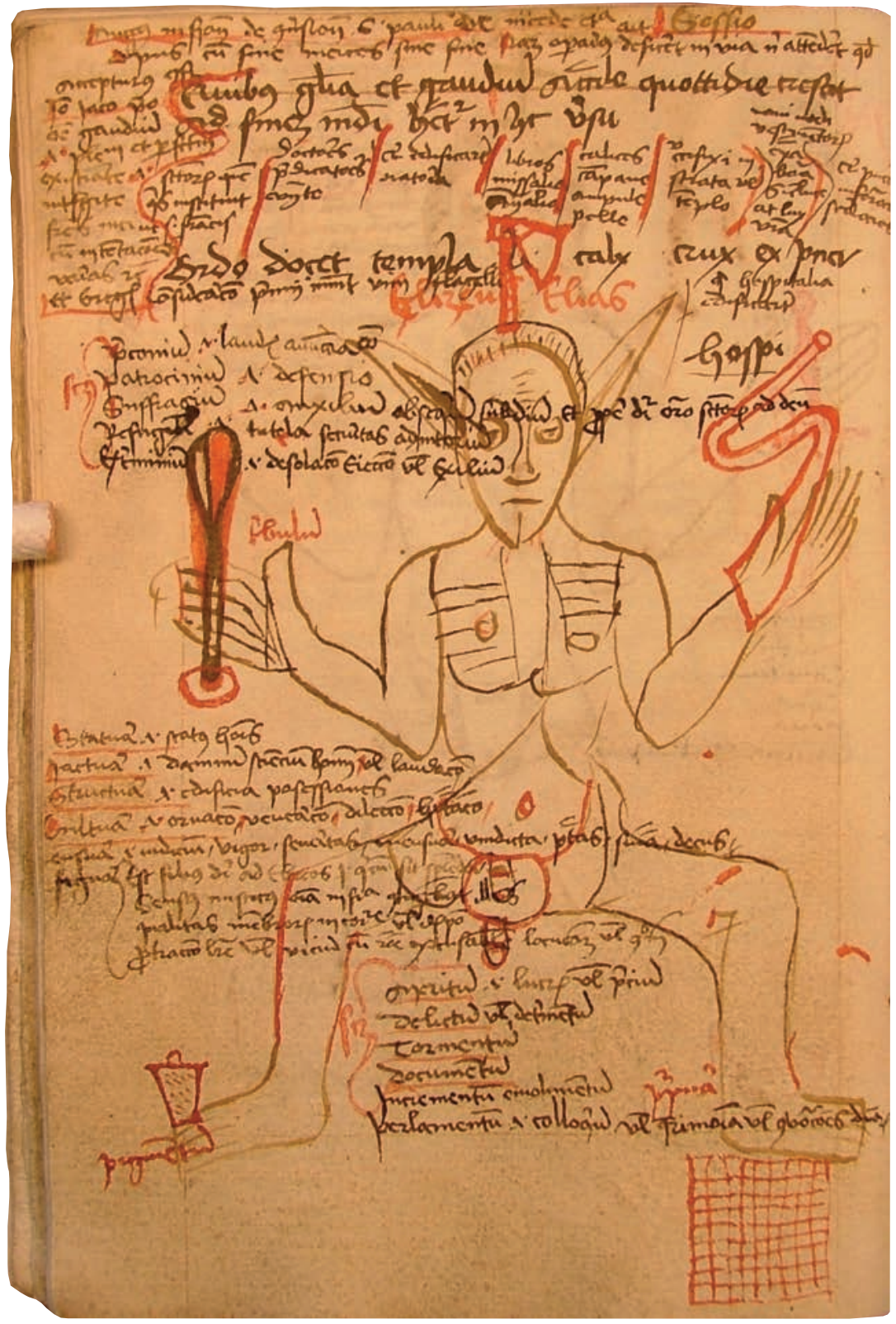

Planche 2. Paulinus de Scalbimiria, Figure pour mémoriser un sermon Kórnik, Bibliothèque de l'Académie, 1122, fol. 24v (avant 1498) 


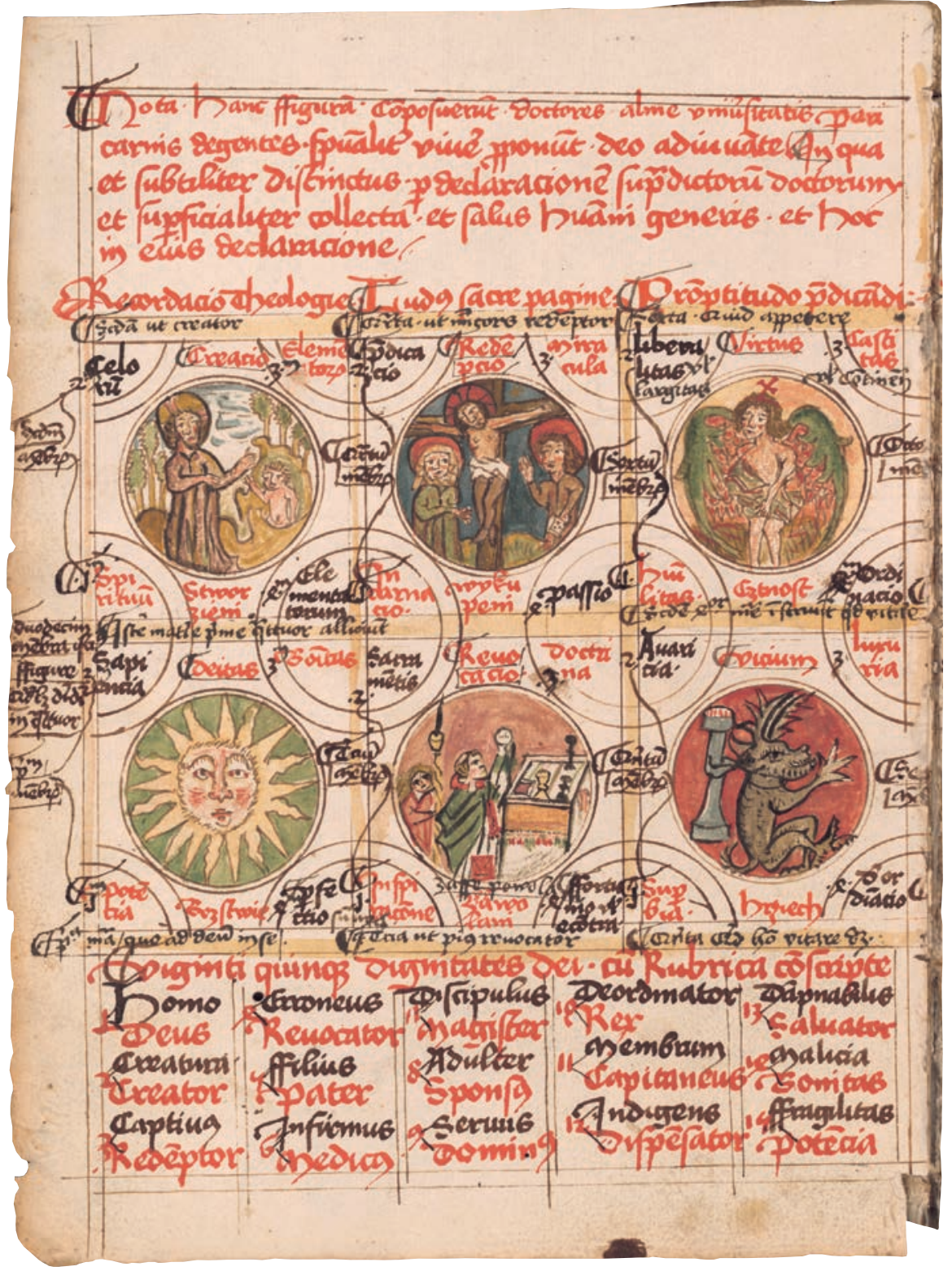

Planche 3. Figure pour la méditation du traité Nota hanc figuram / Pro aliquali intelligentia, dessinée par Ulricus Crux de Telcz (Oldřich Kříž z Telče) Prague, Bibliothèque Nationale, I. G. 11a, fol. 17v-18r (1491) 


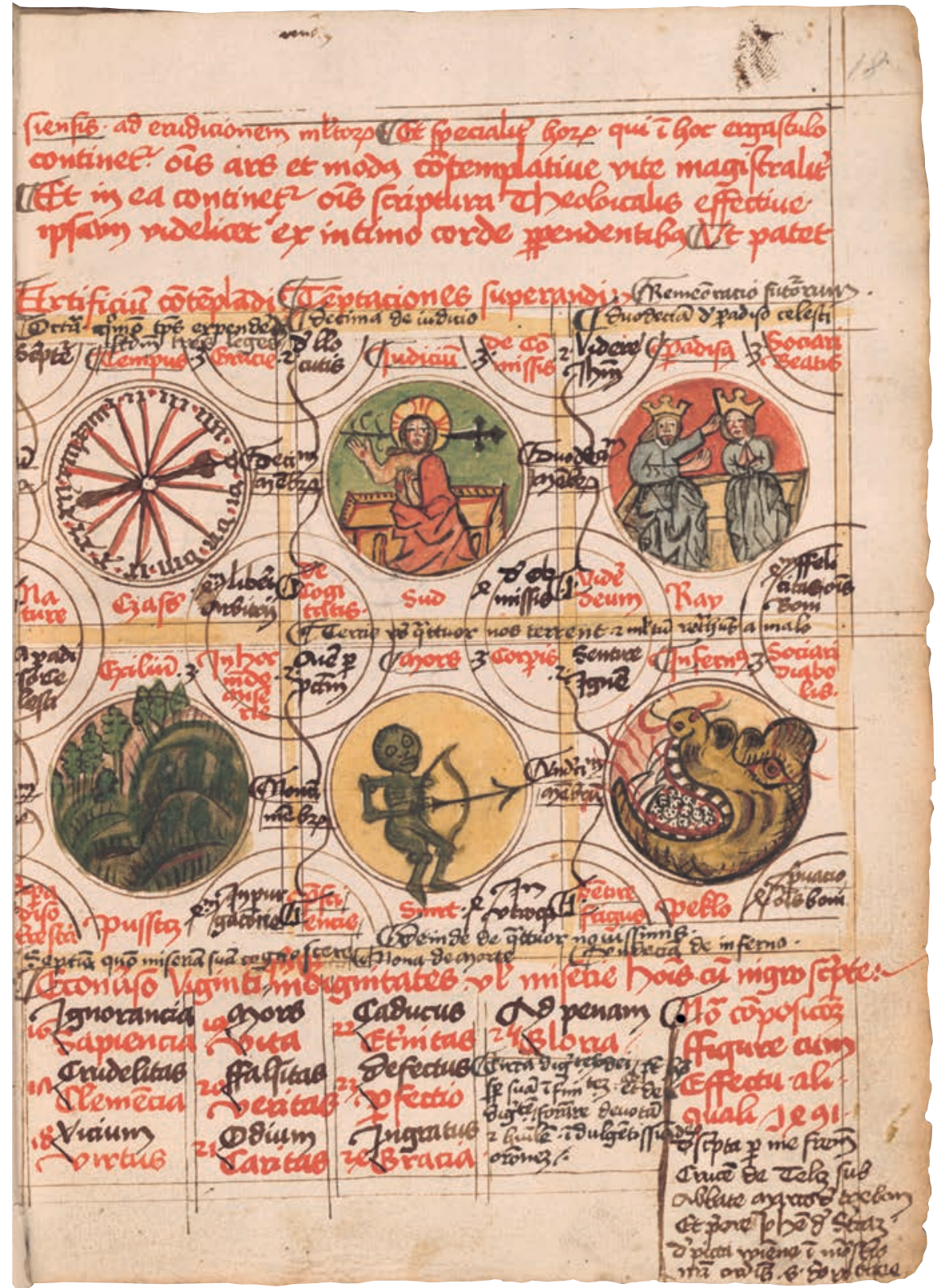

Planche 4. Figure pour la méditation du traité Nota hanc figuram / Pro aliquali intelligentia, dessinée par Ulricus Crux de Telcz (Oldřich Kříž z Telče) Prague, Bibliothèque Nationale, I. G. 11a, fol. 17v-18r (1491) 